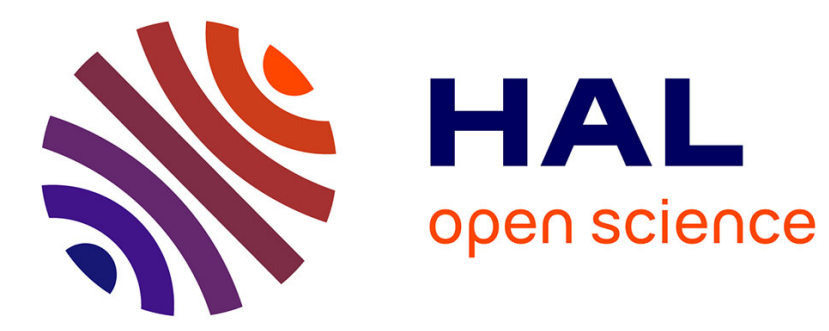

\title{
Performance of piezoelectric shunts for vibration reduction
}

\author{
Olivier Thomas, Julien Ducarne, Jean-François Deü
}

\section{To cite this version:}

Olivier Thomas, Julien Ducarne, Jean-François Deü. Performance of piezoelectric shunts for vibration reduction. Smart Materials and Structures, 2012, 21 (1), pp.015008. 10.1088/09641726/21/1/015008 . hal-01082925

\section{HAL Id: hal-01082925 \\ https://hal.science/hal-01082925}

Submitted on 7 Apr 2015

HAL is a multi-disciplinary open access archive for the deposit and dissemination of scientific research documents, whether they are published or not. The documents may come from teaching and research institutions in France or abroad, or from public or private research centers.
L'archive ouverte pluridisciplinaire HAL, est destinée au dépôt et à la diffusion de documents scientifiques de niveau recherche, publiés ou non, émanant des établissements d'enseignement et de recherche français ou étrangers, des laboratoires publics ou privés. 


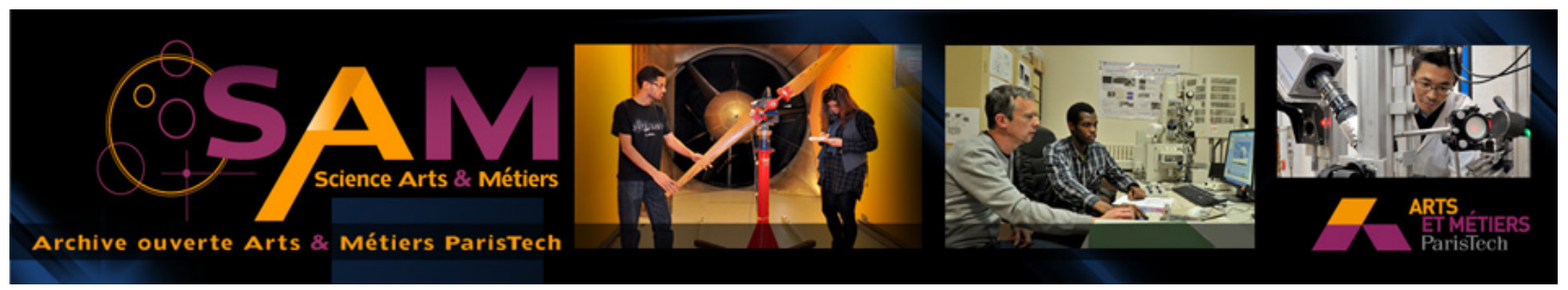

Science Arts \& Métiers (SAM)

is an open access repository that collects the work of Arts et Métiers ParisTech researchers and makes it freely available over the web where possible.

This is an author-deposited version published in: http://sam.ensam.eu Handle ID: .http://hdl.handle.net/10985/8901

\section{To cite this version :}

Olivier THOMAS, Julien DUCARNE, Jean-François DEÜ - Performance of piezoelectric shunts for vibration reduction - Smart Materials and Structures - Vol. 21, n , p.015008 - 2012 


\title{
Performance of piezoelectric shunts for vibration reduction
}

\author{
O Thomas, J Ducarne and J-F Deü
}

Structural Mechanics and Coupled Systems Laboratory, Conservatoire National des Arts et Métiers, 2 Rue Conté, 75003 Paris, France

E-mail: olivier.thomas@cnam.fr

\begin{abstract}
This work addresses passive reduction of structural vibration by means of shunted piezoelectric patches. The two classical resistive and resonant shunt solutions are considered. The main goal of this paper is to give closed-form solutions to systematically estimate the damping performances of the shunts, in the two cases of free and forced vibrations, whatever the elastic host structure is. Then it is carefully demonstrated that the performance of the shunt, in terms of vibration reduction, depends on only one free parameter: the so-called modal electromechanical coupling factor (MEMCF) of the mechanical vibration mode to which the shunts are tuned. Experiments are proposed and an excellent agreement with the model is obtained, thus validating it.
\end{abstract}

(Some figures may appear in colour only in the online journal)

\section{Introduction}

Piezoelectric materials are proposed for many applications, especially in the field of dynamics where their properties of coupling mechanical stress and strain with an electric circuit are used to detect, measure, or control the vibrations. Some of today's active research fields that use piezoelectric materials are energy harvesting, passive or semi-passive structural vibration damping, active vibration control, shape adaptation and structural health monitoring [1]. Piezoelectric materials have also become more widely used in micro/nano electromechanical systems (M/NEMS) as an alternative to the traditional electrostatic transduction technique [2-4].

In this paper, the specific application of passive reduction of structural vibration by means of shunted piezoelectric patches is addressed both theoretically and experimentally. The two simplest shunt circuits are considered: a simple resistor (resistive shunt or R-shunt) or a resistor in series with an inductor (resonant shunt or RL-shunt). Compared to active control techniques, these passive techniques have the advantage of being simple to implement, always stable and not requiring digital signal processors and bulky power amplifiers.

The R- and RL-shunt techniques were proposed by Hagood and Von Flotow in a pioneering paper of the 1990s [5], after an idea proposed in [6]. The two proposed circuits are the electrical analogs of a Lanchester damper for the resistive shunt and a tuned mass damper (or Frahm damper) for the resonant shunt [7]. Since then, several improvements and variations have been proposed. First, several associations (in parallel or in series) of the resistor and the inductor were investigated and compared in [8] and [9]. Since RL-shunt tuning requires a very large inductor (of several tens of henrys) for usual mechanical frequencies, some authors have proposed the use of an additional capacitance to reduce the optimal inductance, also resulting in a reduction of the damping performances [10]. The same technique can be used in an opposite way, by using a negative capacitance, to increase the coupling factor and thus the shunt performances. This technique, however, is active, can be unstable and relies on synthetic electrical components $[11,12]$. Since the effect of a simple RL-shunt circuit is reduced to a narrow frequency band around the mechanical resonance to which it is tuned, several techniques have been proposed to damp several resonances at the same time: a RL-shunt network connected to a single piezoelectric patch in [13], an array of interconnected RL-shunted patches in [14-16] or a periodic array of independent shunted patches in $[17,18]$. To overcome the perfect tuning limitation of the RL-shunt, adaptive circuits, in which the inductor is automatically tuned to the mechanical resonance, have been 
proposed in $[19,20]$. Those passive shunt techniques can also be used to enhance active control techniques [21]. Some recent works have proposed nonclassical piezoelectric patch shapes to enhance the efficiency of the shunt [22]. Finally, some semi-passive techniques, commonly known as 'switch' techniques, have recently been proposed to overcome the above cited drawbacks of the RL-shunt of large inductance and fine tuning. They are based on repeated changes of the electrical impedance synchronously with oscillation of the structure (see [23] and reference therein). Three review articles and a recent book on the subject of adaptive structures may also by cited [1,24-26], as well as a recent application [27].

The main goal of this paper is to systematically evaluate the performances, in term of vibration attenuation, of a either $\mathrm{R}$ - or a RL-shunt connected to a piezoelectric patch glued on a host elastic structure. The vibration reduction brought by the shunt is measured with two indicators: an added damping factor for the free vibration case and a gain reduction in the forced vibration case. Closed-form expressions for those indicators are obtained, which are, to the knowledge of the authors, original. It is shown that those indicators depend only on two parameters: the so-called modal electromechanical coupling factor (MEMCF) and the structural damping. The same result is found for the optimal electrical parameters (the resistor and inductor values) used to tune the shunts on a particular mode. As a consequence, since the structural damping is in most practical cases problem data, the only parameter that has to be considered in the design of the shunt is the MEMCF. In fact, several MEMCFs are defined for a given structure with piezoelectric patches, each one being associated with one piezoelectric patch and one vibration eigenmode of the structure. Here they naturally appear in the equations after expanding the model on the short circuit vibration modes. Those MEMCFs are found very close to the effective electromechanical coupling factor (EEMCF) defined in [28] and their importance has been pointed out by several authors in the past $[5,29,30,8,31]$. Analogous results were also found when investigating switched shunting [23]. One of the quantitative results of the present study is that a resistive shunt can be efficient in terms of vibration reduction for structures that have very low structural damping. An application example is turbojet blades [32]. That is why both resistive and resonant shunts are considered with the same importance in this study.

The outline of the paper is now described. In section 2, a generic electromechanical model, obtained by modal expansion, is proposed. In sections 3 and 4 the R- and RL-shunts are considered. Closed-form expressions for the optimally tuned electrical parameters are obtained in both the free and forced vibration cases. The damping performances are also estimated, which leads again to closed-form relations that can be universally applied since they depend only on the MEMCF and the structural damping. Finally, in section 5, experiments in both free and forced vibration are proposed to validate the strategy. In particular, estimation of the shunt performance as a function of the MEMCF was found to be in excellent agreement with the theory.

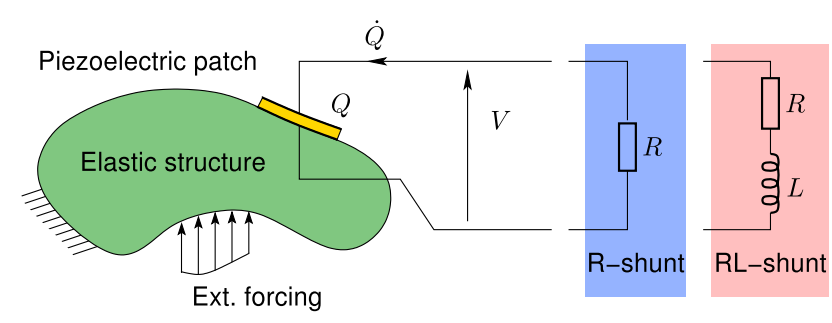

Figure 1. An arbitrary structure with a piezoelectric patch connected to a resistive or a resonant shunt.

\section{Model formulation}

We consider an arbitrary elastic structure with one piezoelectric patch, sketched in figure 1 . We denote by $\boldsymbol{U}(\boldsymbol{x}, t)$ the displacement of any point $\boldsymbol{x}$ of the structure, at time $t$. A resistive or a resonant shunt is connected to the piezoelectric patch; $V$ denotes the voltage between the electrodes, which is also the shunt terminal voltage, and $Q$ is the electric charge in one of the electrodes. Considering the convention of sign for $V$ on figure $1, Q$ is precisely the charge in the upper electrode. Several models for this coupled electromechanical system can be obtained, either in an analytic fashion [33-35] or using a finite-element discretization [36-39]. Then, a reduced order model can be obtained by expanding the displacement $\boldsymbol{U}$ onto $N$ vibration eigenmodes:

$$
\boldsymbol{U}(\boldsymbol{x}, t)=\sum_{i=1}^{N} \boldsymbol{\Phi}_{i}(\boldsymbol{x}) q_{i}(t) .
$$

One can show that the modal coordinates $q_{i}(t)$ are solutions of a problem of the form [39, 35]:

$$
\begin{gathered}
\ddot{q}_{i}+2 \xi_{i} \omega_{i} \dot{q}_{i}+\omega_{i}^{2} q_{i}-\chi_{i} V=F_{i}, \quad \forall i \in\{1 \ldots N\}, \\
C V-Q+\sum_{i=1}^{N} \chi_{i} q_{i}=0 .
\end{gathered}
$$

The electromechanical model of the problem is thus described by $N$ modal equations, corresponding to the balance law of mechanical forces, and one electrical equation, associated with the balance of electric charges on the piezoelectric electrodes. Here, the short circuit eigenmodes are used. They are the vibration modes of the structure with its piezoelectric patch short-circuited $(V=0, \forall p=1, \ldots, P)$. Thus, $\left(\omega_{i}, \boldsymbol{\Phi}_{i}\right)$ denotes the angular natural frequency and mode shape of the corresponding $i$ th mode, respectively. The electromechanical coupling appears in those equations by a modal coupling coefficient $\chi_{i}$, that characterizes the energy transfer between the $i$ th mode shape and the piezoelectric patch. Those coefficients can be computed in a particular problem, either with an analytical model where closed-form solutions are available [35] or by considering a finite-element model [39]. The electric capacitance of the patch is denoted by $C$. Finally, a modal structural damping term, of factor $\xi_{i}$, has been added.

It is convenient to rewrite equation ( $2 a$ ) with $Q$ as the electrical unknown. By introducing equation $(2 b)$ into $(2 a)$ 
to eliminate $V$, one obtains the following set of equations, equivalent to equation $(2 a)$ :

$\ddot{q}_{i}+2 \xi_{i} \omega_{i} \dot{q}_{i}+\omega_{i}^{2} q_{i}-\frac{\chi_{i}}{C} \sum_{j=1}^{N} \chi_{j} q_{j}+\frac{\chi_{i}}{C} Q=F_{i}$,

$$
\forall i \in\{1 \ldots N\}
$$

Those equations can be rewritten to introduce a dimensionless coupling factor $k_{i}$ by using the following change of variables:

$$
\bar{V}=\sqrt{C} V, \quad \bar{Q}=\frac{Q}{\sqrt{C}} .
$$

Note that $\bar{V}$ and $\bar{Q}$ are not dimensionless. Using equations (3) and $(2 b)$, the modal electromechanical problem is then written:

$$
\begin{gathered}
\ddot{q}_{i}+2 \xi_{i} \omega_{i} \dot{q}_{i}+\omega_{i}^{2} q_{i}+\omega_{i} k_{i} \sum_{j=1}^{N} \omega_{j} k_{j} q_{j}-\omega_{i} k_{i} \bar{Q}=F_{i}, \\
\bar{V}-\bar{Q}+\sum_{i=1}^{N} \omega_{i} k_{i} q_{i}=0 .
\end{gathered}
$$

The dimensionless modal electromechanical coupling factor $k_{i}$ has been introduced and is defined by:

$$
k_{i}=\frac{\chi_{i}}{\omega_{i} \sqrt{C}}
$$

Its physical meaning is as follows. If the above model is truncated to its $j$ th one mode only $\left(q_{i}=0 \forall i \neq j\right)$, equation $(5 b)$ becomes:

$$
\ddot{q}_{j}+2 \xi_{j} \omega_{j} \dot{q}_{j}+\hat{\omega}_{j}^{2} q_{j}-\omega_{j} k_{j} \bar{Q}=F_{j},
$$

where

$$
\hat{\omega}_{j}=\omega_{j} \sqrt{1+k_{j}^{2}}
$$

The above defined frequency $\hat{\omega}_{j}$ is the natural frequency of the mechanical oscillator (7) with $\bar{Q}=0$. As a consequence, $\hat{\omega}$ is an approximation of the $j$ th system's natural frequency in open circuit as long as the natural frequencies are far enough from one another, so that the single mode approximation is justified. If we denote by $\omega_{j}^{\mathrm{SC}}$ and $\omega_{j}^{\mathrm{OC}}$ the $j$ th natural frequencies of the electromechanical system respectively in short circuit (SC) and open circuit (OC) conditions, we have $\omega_{j}=\omega_{j}^{\mathrm{SC}}$ (by definition of $\omega_{j}$ ) and $\hat{\omega}_{j}=\omega_{j}^{\mathrm{OC}}$. Then, equation (8) shows that the MEMCF $k_{j}$ is close, in absolute value, to the $j$ th effective coupling factor $k_{\mathrm{eff}, j}[28]$ :

$$
\left|k_{j}\right| \simeq k_{\mathrm{eff}, j}=\sqrt{\frac{\left(\omega_{j}^{\mathrm{OC}}\right)^{2}-\left(\omega_{j}^{\mathrm{SC}}\right)^{2}}{\left(\omega_{j}^{\mathrm{SC}}\right)^{2}}} .
$$

This formula is convenient in practice, since $\omega_{j}^{\mathrm{OC}}$ and $\omega_{j}^{\mathrm{SC}}$ can be easily measured, to obtain a good approximation of $\left|k_{j}\right|$. For more details, the reader can refer to [39, 23, 35].

The above formulation can be extended to a network of patches connected in series or in parallel to a single shunt circuit, giving an equivalent coupling factor $k_{j}$ that measures the energy transfers between the electric circuit and the $j$ th mode through the electric network. Examples of two collocated patches are given in $[39,35]$ and used in section 5 of the present paper.

\section{Resistive shunt}

The case of a resistive shunt, where the electric circuit connected to the piezoelectric patches is simply a resistance of constant $R$, is considered in this section (figure 1). In this case, the relationship between $V$ and $Q$ is simply given by Ohm's law, $V=-R \dot{Q}$. Then, using the reduced parameters of equations (4) leads to the replacement of equation $(5 b)$ by

$$
\tau_{\mathrm{e}} \dot{\bar{Q}}+\bar{Q}-\sum_{i=1}^{N} \omega_{i} k_{i} q_{i}=0
$$

where the resistive shunt time constant is

$$
\tau_{\mathrm{e}}=R C .
$$

In the following, the shunt optimization and performance estimation will be done by considering only one structural mode. Thus, the $N$ degrees of freedom model of equations $(5 a)$ and (10) is truncated to the $j$ th mechanical mode (i.e. $q_{i} \equiv 0, \forall i \neq$ j):

$$
\begin{gathered}
\ddot{q}_{j}+2 \xi_{j} \omega_{j} \dot{q}_{j}+\hat{\omega}_{j}^{2} q_{j}-\omega_{j} k_{j} \bar{Q}=F_{j}, \\
\tau_{\mathrm{e}} \dot{\bar{Q}}+\bar{Q}-\omega_{j} k_{j} q_{j}=0 .
\end{gathered}
$$

\section{1. $R$-shunt free response}

\subsubsection{R-shunt optimal tuning: pole placement technique.}

The so-called pole placement technique (PP) [5] is used here to optimize the vibration reduction in the time domain. It consists in finding the value $\tau_{\mathrm{e}}^{\mathrm{PP}}$ of $\tau_{\mathrm{e}}$ that minimizes the time decay of $q_{j}$ and $Q$. One has to maximize the real parts of the three poles of the transfer function associated with equations (12a) and (12b) in free oscillation (i.e. with $\left.F_{j} \equiv 0\right)$. A numerical solution shows that one pole is real negative and the two others are complex conjugates, which allows us to denote them by $\lambda_{1}=-\sigma$ and $\lambda_{2,3}=-\mu \pm \mathrm{j} \omega$, where $\sigma, \mu$ and $\omega$ are three real positive numbers. One has thus to maximize $\sigma$ and $\mu$. It can be numerically shown that $\sigma$ is about 1000 times higher than $\mu$, for various values of $\tau_{\mathrm{e}}$. The consequence is that the time decay of $q_{j}(t)$ and $Q(t)$ is mainly governed by $\exp (-\mu t)$. As a consequence, the PP criterion consists in finding the value of $\tau_{\mathrm{e}}$ that maximizes $\mu$.

An analytical estimation of $\tau_{\mathrm{e}}^{\mathrm{PP}}$ can be obtained by neglecting the structural damping $\xi_{j}[5,8]$. By imposing $\partial \mu / \partial \tau_{\mathrm{e}}=0$, one obtains the following optimal value for $\tau_{\mathrm{e}}$ (see appendix A.1):

$$
\tau_{\mathrm{e}}^{\mathrm{PP}}=\frac{1}{\omega_{j}\left(1+k_{j}^{2} / 2\right)} .
$$

The corresponding values for $\sigma, \mu$ and $\omega$ are

$$
\begin{gathered}
\sigma^{\mathrm{R}}=\omega_{j}, \quad \mu^{\mathrm{R}}=\omega_{j} k_{j}^{2} / 4, \\
\omega^{\mathrm{R}}=\omega_{j} \sqrt{1+k_{j}^{2} / 2-k_{j}^{4} / 16 .}
\end{gathered}
$$




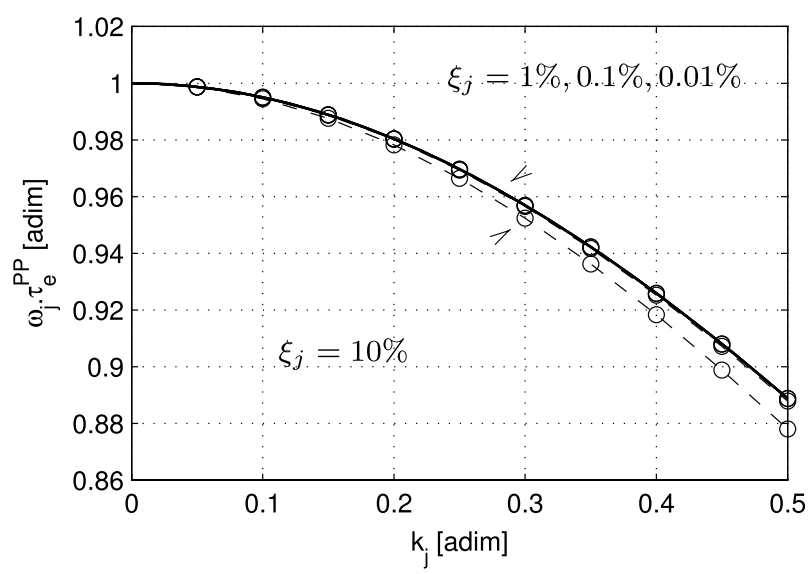

Figure 2. Optimal value of the dimensionless electric time constant $\tau_{\mathrm{e}}^{\mathrm{PP}}$, for resistive shunt, as a function of coupling factor $k_{j}$. The quantity $\tau_{\mathrm{e}}^{\mathrm{PP}} \omega_{j}$ is shown instead of $\tau_{\mathrm{e}}^{\mathrm{PP}}$. '-'. value of equation (13) with no structural damping $\xi_{j}$; ' $-\circ-$ ', numerical values $\tau_{\mathrm{e}}^{\text {PPnum }}$ for several values of the structural damping $\xi_{j}$ (for $\xi_{j} \in\{1 \%, 0.1 \%$, $0.01 \%$, all curves are merged).

One can verify that, as stated above, for usual values of $k_{j}$ (small as compared to 1 ), we have $\sigma^{\mathrm{R}} \gg \mu^{\mathrm{R}}$.

If the structural damping is not neglected, we have numerically estimated the value $\tau_{\mathrm{e}}^{\mathrm{PPnum}}$ that maximizes $\mu$ (by computing the poles of equations (12a) and (12b) with $\xi_{j} \neq 0$ ), for several values of $\omega_{j}, k_{j}$ and $\xi_{j}$. Figure 2 shows that for small values of $\xi_{j}$ (for $\xi_{j}<1 \%$ ), no significant differences are obtained between $\tau_{\mathrm{e}}^{\mathrm{PPnum}}$ and $\tau_{\mathrm{e}}^{\mathrm{PP}}$, so that the optimal value of $\tau_{\mathrm{e}}$ with the PP criterion, for $\xi_{j}<1 \%$, is $\tau_{\mathrm{e}}^{\mathrm{PP}}$ given by equation (13).

One can remark that if $k_{j}$ is small compared to 1 ,

$$
1+k_{j}^{2} / 2 \simeq \sqrt{1+k_{j}^{2}} \Longrightarrow 1 / \tau_{\mathrm{e}}^{\mathrm{PP}} \simeq \omega_{j} \sqrt{1+k_{j}^{2}}=\hat{\omega}_{j} .
$$

From a physical point of view, it means that the optimal value $\tau_{\mathrm{e}}$ of the electric time constant is close to the inverse of the open circuit angular frequency.

3.1.2. Vibration reduction estimation. Since, as explained above, the system's free oscillations are mainly governed by the term $\exp (-\mu t), \mu$ measures the total damping of the system, including both structural damping and that due to the resistive shunt. We use as a shunt performance indicator the following damping factor, defined by $\xi_{\text {tot }}^{\mathrm{R}}=\mu^{\mathrm{R}} / \omega^{\mathrm{R}}$. In the case of no structural damping $\left(\xi_{j}=0\right), \xi_{\text {tot }}^{\mathrm{R}}$ takes the form (see equations (14))

$$
\left.\xi_{\mathrm{tot}}^{\mathrm{R}}\right|_{\xi_{j}=0}=\xi_{0}^{\mathrm{R}}=\frac{k_{j}^{2}}{4 \sqrt{1+k_{j}^{2} / 2-k_{j}^{4} / 16}} \simeq \frac{k_{j}^{2}}{4} .
$$

To consider the case of non-zero structural damping, we define the added damping factor by subtracting the structural damping from the total damping: $\xi_{\text {add }}^{\mathrm{R}}=\xi_{\text {tot }}^{\mathrm{R}}-\xi_{j}$. Figure 3 shows $\xi_{\text {add }}^{\mathrm{R}}$ as a function of $k_{j}$, for several values of structural damping $\xi_{j} . \xi_{\text {add }}^{\mathrm{R}}$ is numerically evaluated by computing the poles of equations (12a) and (12b) with $\tau_{\mathrm{e}}=\tau_{\mathrm{e}}^{\text {PPnum }}$. Since

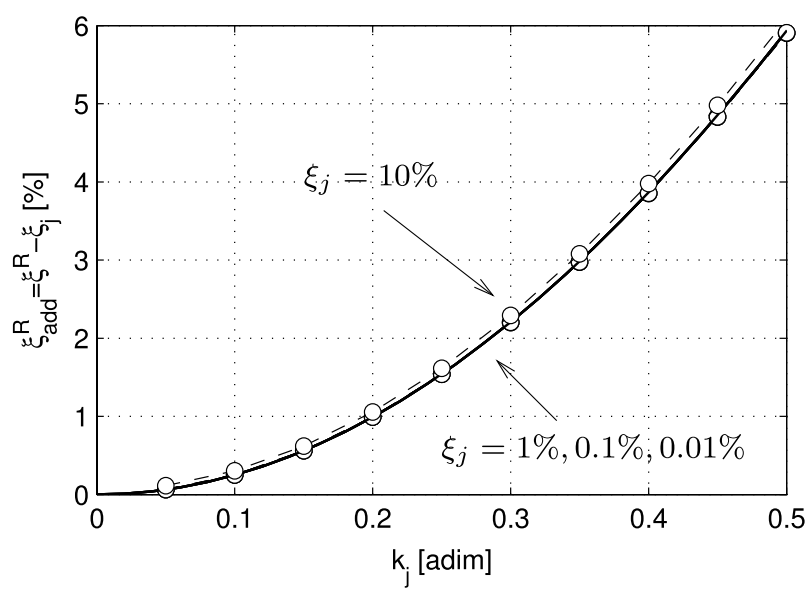

Figure 3. Added damping $\xi_{\text {add }}^{\mathrm{R}}$ in the case of a resistive shunt, for several values of structural damping $\xi_{j}$ (for $\xi_{j} \in\{1 \%, 0.1 \%$, $0.01 \%$, all curves are merged). ',,$- \xi_{\text {add }}^{\mathrm{R}}=\xi_{0}^{\mathrm{R}}$ of equation (16); ' $-\circ-, \xi_{\text {add }}^{\mathrm{R}}$ is numerically computed with $\tau_{\mathrm{e}}=\tau_{\mathrm{e}}^{\text {PPnum }}$.

all curves are almost merged into a single one, we can verify that $\tau_{\mathrm{e}}^{\mathrm{PP}}$ remains a good estimation for the optimal electric time constant, for $\xi_{j}$ up to $10 \%$. This last result enhances the validity range of $\tau_{\mathrm{e}}^{\mathrm{pp}}$, reduced to $\xi_{j}<1 \%$ at the end of section 3.1.1.

Moreover, this shows that the added damping $\xi_{\text {add }}^{\mathrm{R}}$ does not depend on the structural damping $\xi_{\text {, }}$, so that it is perfectly known by its value with no structural damping: $\xi_{\mathrm{add}}^{\mathrm{R}} \simeq \xi_{0}^{\mathrm{R}}$. As a consequence, the total damping with resistive shunt is known analytically by the following formula (valid for $\xi_{j}<$ $10 \%)$ :

$$
\xi_{\text {tot }}^{\mathrm{R}}=\xi_{j}+\xi_{\mathrm{add}}^{\mathrm{R}} \simeq \xi_{j}+\xi_{0}^{\mathrm{R}}
$$

with $\xi_{0}^{\mathrm{R}}$ defined by equation (16).

The above results show that the damping effect of the resistive shunt is significant only for low structural damping. For instance, if $k_{j}=0.15, \xi_{\text {add }}^{\mathrm{R}} \simeq 0.6 \%$, so that $\xi_{\text {tot }}^{\mathrm{R}} \simeq 0.6+$ $\xi_{j}(\%)$. For $\xi_{j}$ of the order of $10 \%, \xi_{\mathrm{tot}}^{\mathrm{R}} \simeq \xi_{j}$ and the effect of the resistive shunt is negligible.

\section{2. $R$-shunt forced response}

\subsubsection{R-shunt optimal tuning: transfer function technique.}

In the case of forced vibration, the so-called transfer function (TF) criterion [5] is used. It consists of choosing the value of the shunt electric resistance $R$ (corresponding to dimensionless parameter $\tau_{\mathrm{e}}$ ) that minimizes the peak amplitude of the frequency response function (FRF) modulus. The frequency response function displacement/force (under harmonic forcing of frequency $\Omega$ ), associated with equations $(12 a)$ and $(12 b)$, is written as

$$
\begin{aligned}
& H(\Omega)= \\
& \quad \frac{1+\mathrm{j} \tau_{\mathrm{e}} \Omega}{\omega_{j}^{2}-\left(1+2 \tau_{\mathrm{e}} \xi_{j} \omega_{j}\right) \Omega^{2}+\mathrm{j} \Omega\left(\tau_{\mathrm{e}} \hat{\omega}_{j}^{2}+2 \xi_{j} \omega_{j}-\tau_{\mathrm{e}} \Omega^{2}\right)} .
\end{aligned}
$$



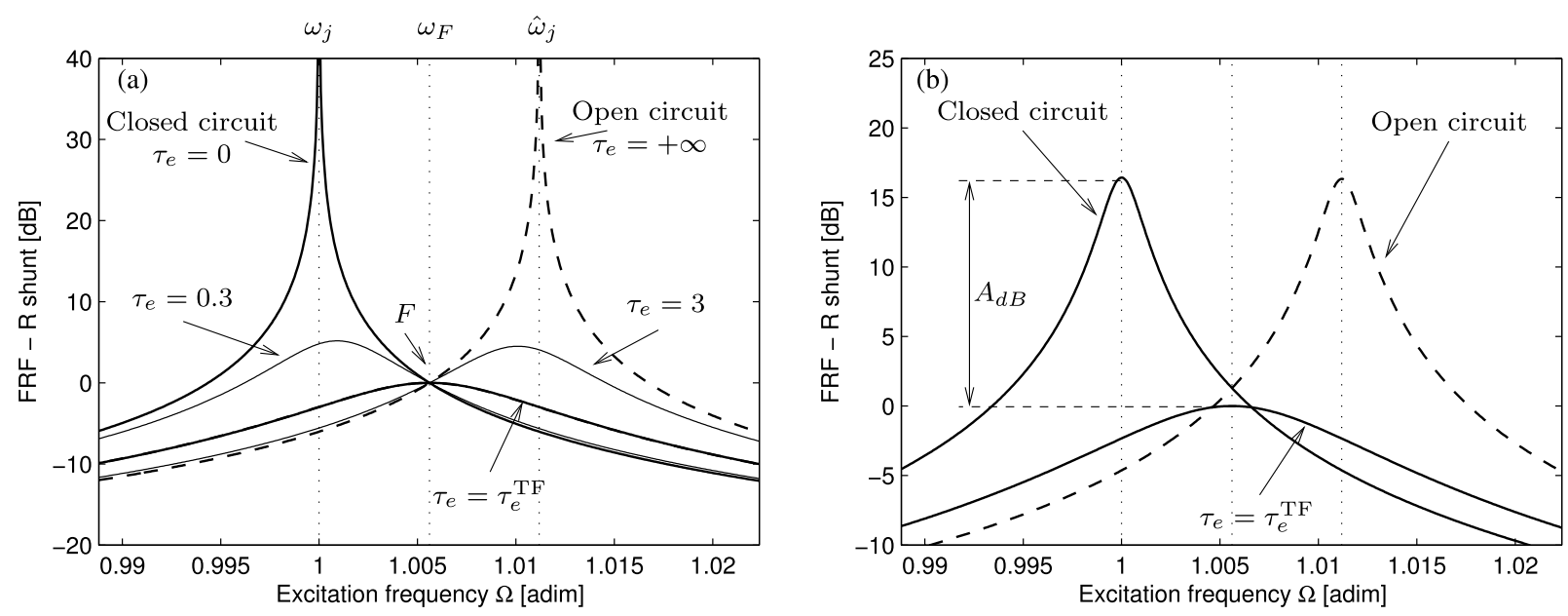

Figure 4. Frequency response function with a resistive shunt for several values of the electric time constant $\tau_{\mathrm{e}}$ (i.e. for various resistances $R$ ): (a) $\xi_{j}=0$ : with no structural damping; (b) with structural damping $\xi_{j}=0.1 \%$.

As suggested in $[5,7,40]$, the optimization problem is solved by exploiting the fact that if the structural damping $\xi_{j}$ is neglected, there exists a point $F$ that is common to all gain curves $|H(\Omega)|$ when $\tau_{\mathrm{e}}$ is varied (see figure $4(\mathrm{a})$ ). The coordinates $\left(\omega_{F}, H_{F}\right)$ of $F$ can be obtained by remarking that among all possible gain curves, it is common to two particular ones: the one in short circuit $\left(R=0 \Rightarrow \tau_{\mathrm{e}}=0\right)$ and the one in open circuit $\left(R=+\infty \Rightarrow \tau_{\mathrm{e}}=+\infty\right)$, for which $|H(\Omega)|$ has simple mathematical forms. Imposing $\left|H\left(\omega_{F}\right)\right|_{\tau_{\mathrm{e}}=0}=$ $\left|H\left(\omega_{F}\right)\right|_{\tau_{\mathrm{e}}=+\infty}$ leads to

$$
\omega_{F}=\omega_{j} \sqrt{1+k_{j}^{2} / 2}, \quad H_{F}=\frac{2}{\omega_{j}^{2} k_{j}^{2}} .
$$

Then, since $F$ is common to all gain curves, the optimum one (the one that has the lowest peak amplitude) has its peak at point $F$. The corresponding optimum value $\tau_{\mathrm{e}}^{\mathrm{TF}}$ of $\tau_{\mathrm{e}}$ with this transfer function criterion thus verifies

$$
\frac{\partial}{\partial \Omega}|H(\Omega)|_{\Omega=\omega_{F}}^{2}=0 .
$$

Evaluating the above equation leads to

$$
\tau_{\mathrm{e}}^{\mathrm{TF}}=\frac{1}{\omega_{F}}=\frac{1}{\omega_{j} \sqrt{1+k_{j}^{2} / 2}} .
$$

Since the above value is valid with no structural damping, we have numerically computed the value $\tau_{\mathrm{e}}^{\text {TFnum }}$ that minimizes the peak amplitude of $|H(\Omega)|$ in the case $\xi_{j} \neq$ 0 . We have shown that no significant difference is obtained between $\tau_{\mathrm{e}}^{\mathrm{TF}}$ and $\tau_{\mathrm{e}}^{\mathrm{TFnum}}$ as long as the structural damping is small (lower than $10 \%$ ), so that the optimal value with the transfer function criterion, for any value of $\xi_{j}$, is $\tau_{\mathrm{e}}^{\mathrm{TF}}$ given by equation (21).

3.2.2. Vibration reduction estimation. To estimate the vibration reduction brought by the resistive shunt, we define the following quantity, referred to as the attenuation (in decibels), by

$$
A_{\mathrm{dB}}=20 \log \frac{H_{\mathrm{SC}}}{H_{\text {shunt }}}
$$

where $H_{\mathrm{SC}}$ is the FRF peak amplitude in short circuit situation and $H_{\text {shunt }}$ is the one with the shunt connected to the piezoelectric patches. It represents the reduction of the vibration level brought about by the shunt, with reference to the short circuit situation (see figure 4(b)).

In the short circuit situation, the FRF amplitude at the resonance is

$$
H_{\mathrm{SC}}=\left|H\left(\tilde{\omega}_{j}\right)\right|_{\tau_{\mathrm{e}}=0}=\frac{1}{2 \xi_{j} \omega_{j}^{2}} \frac{1}{\sqrt{1-\xi_{j}^{2}}},
$$

where $\tilde{\omega}_{j}=\omega_{j} \sqrt{1-2 \xi_{j}^{2}}$ is the resonance frequency in short circuit.

With non-zero structural damping, the gain curves no longer cross at point $F$ (see figure 4(b)). However, if $\xi_{j}$ is small, the location of the optimal gain curve (the one which has the lowest peak amplitude) is very close to the one with $\xi_{j}=0$ and its peak is thus very close to point $F$. Consequently, a good estimation of the gain curve peak amplitude with the shunt optimally tuned is then obtained by the gain at point $F: H_{\mathrm{opt}}=\left|H\left(\omega_{F}\right)\right|$ with $\tau_{\mathrm{e}}=\tau_{\mathrm{e}}^{\mathrm{TF}}$. After a few mathematical developments, the maximal attenuation brought by the resistive shunt can be written as

$$
\begin{aligned}
& A_{\mathrm{dB}}^{\mathrm{R}}=20 \log \frac{H_{\mathrm{SC}}}{H_{\mathrm{opt}}} \simeq 20 \log \frac{H_{\mathrm{SC}}}{\left|H\left(\omega_{F}\right)\right|_{\tau_{\mathrm{e}}=\tau_{\mathrm{e}}^{\mathrm{TF}}}} \\
& =20 \log \frac{k_{j}^{2}+2 \sqrt{2} \xi_{j} \sqrt{2+k_{j}^{2}}}{4 \xi_{j} \sqrt{1-\xi_{j}^{2}}} .
\end{aligned}
$$

The approximation used to obtain the above analytical result is now verified, by numerically computing $H_{\mathrm{opt}}$ as the maximum value of $|H(\Omega)|$ as a function of $\Omega$, with $\tau_{\mathrm{e}}$ adjusted to its optimal numerical value $\tau_{\mathrm{e}}^{\mathrm{TFnum}}$. Since no noticeable difference is observed for $\xi_{j}<10 \%$ (see figure 5), the level 


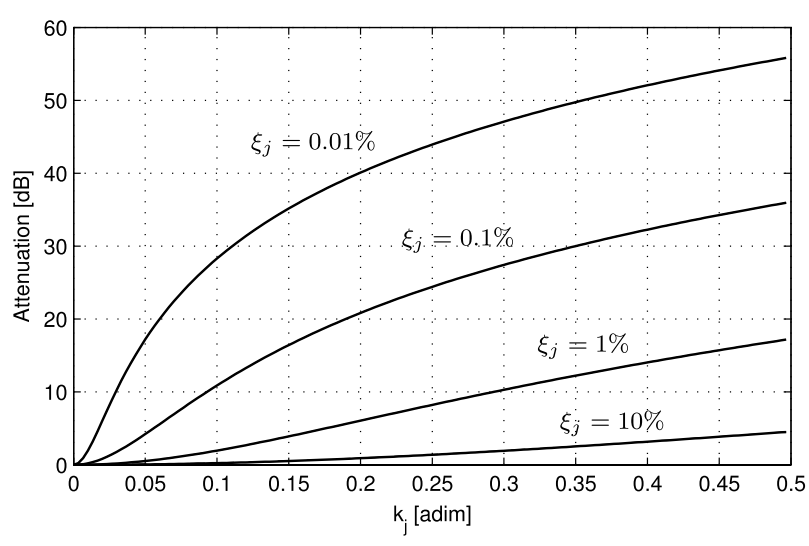

Figure 5. Attenuation $A_{\mathrm{dB}}^{\mathrm{R}}$ due to a resistive shunt as a function of coupling factor $k_{j}$, for several values of structural damping $\xi_{j}$. For each value of $\xi_{j}$, two curves are merged: the one of equation (24) and the one numerically computed with $\tau_{\mathrm{e}}=\tau_{\mathrm{e}}^{\mathrm{TFnum}}$.

of attenuation of vibration due to the resistive shunt is given by equation (24).

The above equation (24) is useful to evaluate the performance of a resistive shunt, that depends only on the modal coupling factor $k_{j}$ and the structural damping $\xi_{j}$. Figure 5 shows $A_{\mathrm{dB}}^{\mathrm{R}}$ as a function of $k_{j}$ for several values of $\xi_{j}$. In the same way as in the case of the free response (section 3.1.2), the effect of the resistive shunt is significant only for low structural damping. For instance, if $k_{j}=0.15$, up to $35 \mathrm{~dB}$ of attenuation are obtained with structural damping of $\xi_{j}=0.01 \%$ whereas only $0.5 \mathrm{~dB}$ are observed with $\xi_{j}=$ $10 \%$. Moreover, one can remark that $A_{\mathrm{dB}}^{\mathrm{R}}$ does not tends to zero when the coupling coefficient $k_{j}$ tends to zero. This is a consequence of the definition of $A_{\mathrm{dB}}^{\mathrm{R}}: H_{\mathrm{opt}}$ is the gain at the frequency $\omega_{F}$, which tends to natural frequency $\omega_{j}$, which slightly differs from the resonance frequency $\tilde{\omega}_{j}$ used to calculate $H_{\mathrm{SC}}$ (equations (23) and (24)).

\section{Resonant shunt}

The case of a resonant shunt, where the electric circuit connected to the piezoelectric patches is a resistance $R$ and an inductance $L$ in series, is considered in this section (figure 1). In this case, the relationship between $V$ and $Q$ is $V=-R \dot{Q}-$ $L \ddot{Q}$. Then, using the reduced parameters of equations (4) leads to the replacement of equation $(5 b)$ by

$$
\frac{1}{\omega_{\mathrm{e}}^{2}} \ddot{\bar{Q}}+\frac{2 \xi_{\mathrm{e}}}{\omega_{\mathrm{e}}} \dot{\bar{Q}}+\bar{Q}-\sum_{i=1}^{N} \omega_{i} k_{i} q_{i}=0,
$$

where the resonant shunt frequency $\omega_{\mathrm{e}}$ and damping factor $\xi_{\mathrm{e}}$ are defined by

$$
\omega_{\mathrm{e}}=\frac{1}{\sqrt{L C}}, \quad \xi_{\mathrm{e}}=\frac{R C \omega_{\mathrm{e}}}{2}=\frac{R}{2} \sqrt{\frac{C}{L}} .
$$

In the following, shunt optimization and performance estimation will be done by considering only one structural mode. Thus, the $N$ degrees of freedom model of equations $(5 a)$ and $(25)$ is truncated to the $j$ th mechanical mode

$$
\begin{aligned}
& \text { (i.e. } \left.q_{i} \equiv 0, \forall i \neq j\right) \text { : } \\
& \qquad \begin{aligned}
\ddot{q}_{j}+2 \xi_{j} \omega_{j} \dot{q}_{j}+\hat{\omega}_{j}^{2} q_{j}-\omega_{j} k_{j} \bar{Q} & =F_{j}, \\
& \frac{1}{\omega_{\mathrm{e}}^{2}} \ddot{\bar{Q}}+\frac{2 \xi_{\mathrm{e}}}{\omega_{\mathrm{e}}} \dot{\bar{Q}}+\bar{Q}-\omega_{j} k_{j} q_{j}=0 .
\end{aligned}
\end{aligned}
$$

\subsection{RL-shunt free response}

4.1.1. RL-shunt optimal tuning: pole placement technique. As in the case of a resistive shunt (section 4.1.1), the pole placement (PP) technique is used here to optimize the vibration reduction in the time domain. It consists in finding the values $\left(\omega_{\mathrm{e}}^{\mathrm{PP}}, \xi_{\mathrm{e}}^{\mathrm{PP}}\right)$ of $\left(\omega_{\mathrm{e}}, \xi_{\mathrm{e}}\right)$ that minimize the time decay of $q_{j}$ and $Q$. By numerically computing the four poles $\lambda_{i}$ of the transfer function of equations $(27 a)$ and (27b), it can be shown that their real parts have a maximum absolute value when the four poles degenerate to two complex conjugates, denoted here by $\lambda_{1}=\lambda_{2}=-\mu+\mathrm{j} \omega$ and $\lambda_{3}=\lambda_{4}=-\mu-$ $\mathrm{j} \omega$. An analytical estimation of $\left(\omega_{\mathrm{e}}^{\mathrm{PP}}, \xi_{\mathrm{e}}^{\mathrm{PP}}\right)$ can be obtained by neglecting the structural damping $\xi_{j}$. One obtains (see appendix A.2):

$$
\omega_{\mathrm{e}}^{\mathrm{PP}}=\omega_{j}\left(1+k_{j}^{2}\right), \quad \xi_{\mathrm{e}}^{\mathrm{PP}}=\frac{\left|k_{j}\right|}{\sqrt{1+k_{j}^{2}}} .
$$

The corresponding values for $\mu$ and $\omega$ are

$$
\mu^{\mathrm{RL}}=\frac{\left|k_{j}\right| \hat{\omega}_{j}}{2}, \quad \omega^{\mathrm{RL}}=\hat{\omega}_{j} \sqrt{1-k_{j}^{2} / 4} .
$$

If the structural damping is not neglected, one can numerically estimate the values $\omega_{\mathrm{e}}^{\text {PPnum }}$ and $\xi_{\mathrm{e}}^{\text {PPnum that }}$ maximize $\mu$ (by computing the transfer function poles with $\xi_{j} \neq 0$ ), for several values of $\omega_{j}, k_{j}$ and $\xi_{j}$. For small values of $\xi_{j}$ (for $\xi_{j}<1 \%$ ), no significant differences are obtained between $\left(\omega_{\mathrm{e}}^{\text {PPnum }}, \xi_{\mathrm{e}}^{\text {PPnum }}\right)$ and $\left(\omega_{\mathrm{e}}^{\mathrm{PP}}, \xi_{\mathrm{e}}^{\mathrm{PP}}\right)$, as shown on figure 6. Consequently, the optimal values of $\omega_{\mathrm{e}}$ and $\xi_{\mathrm{e}}$ with the PP criterion, for $\xi_{j}<1 \%$, are $\omega_{\mathrm{e}}^{\mathrm{PP}}$ and $\xi_{\mathrm{e}}^{\mathrm{PP}}$ given by equation (28). In the same manner as in the resistive shunt case, one can remark that if $k_{j}$ is small compared to $1, \omega_{\mathrm{e}}^{\mathrm{PP}} \simeq$ $\omega_{j}$ and $\xi_{\mathrm{e}}^{\mathrm{PP}} \simeq\left|k_{j}\right|$. The optimal value of the electric frequency is close to the mechanical natural frequency and the optimal value of the electric damping factor is close to the coupling factor.

4.1.2. Estimation of vibration reduction. As shown before, all transfer function poles have the same real part $-\mu$. As in the case of a resistive shunt, we use as a shunt performance indicator the following damping factor, defined by $\xi_{\text {tot }}^{\mathrm{RL}}=$ $\mu^{\mathrm{RL}} / \omega^{\mathrm{RL}}$. In the case of no structural damping $\left(\xi_{j}=0\right), \xi_{\text {tot }}^{\mathrm{RL}}$ takes the form (see equations (29))

$$
\left.\xi_{\mathrm{tot}}^{\mathrm{RL}}\right|_{\xi_{j}=0}=\xi_{0}^{\mathrm{RL}}=\frac{\left|k_{j}\right|}{\sqrt{4-k_{j}^{2}}} \simeq\left|k_{j}\right| / 2 .
$$

Again, the case of non-zero structural damping is considered by defining the added damping factor $\xi_{\text {add }}^{\mathrm{RL}}=\xi_{\text {tot }}^{\mathrm{RL}}-\xi_{j}$. Figure 7 shows $\xi_{\text {add }}^{\mathrm{RL}}$ as a function of $k_{j}$, for several values of structural damping $\xi_{j}$. $\xi_{\text {add }}^{\mathrm{RL}}$ is numerically evaluated by computing 

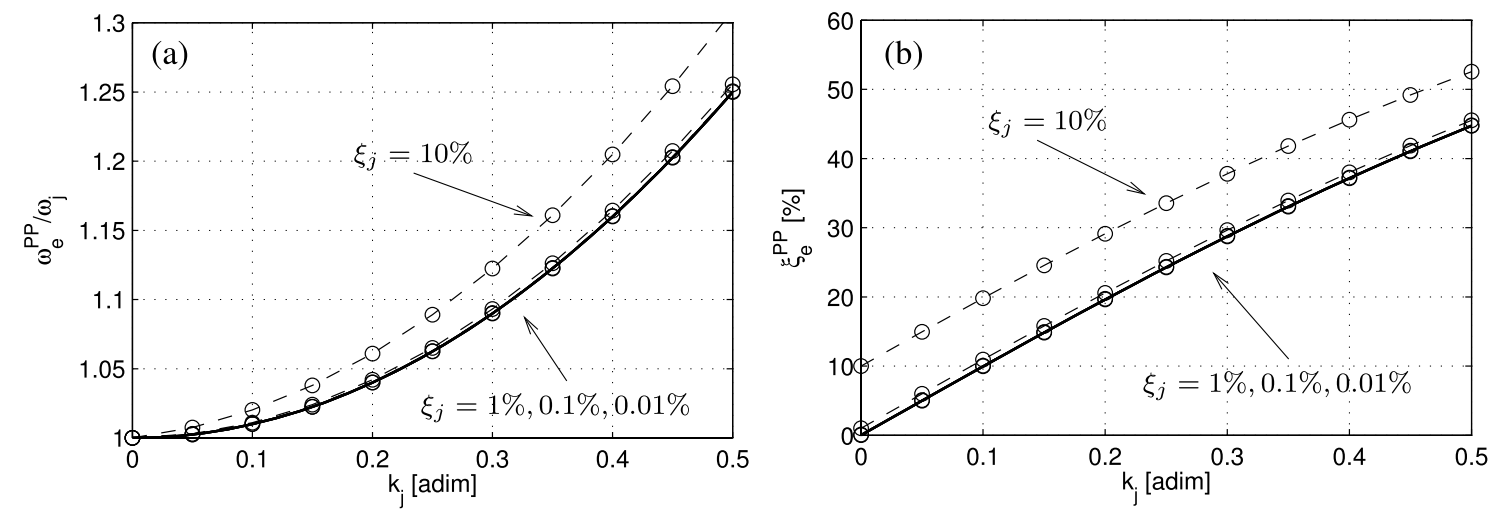

Figure 6. (a) Optimal value of the dimensionless electric frequency $\omega_{\mathrm{e}}^{\mathrm{PP}}$ for resonant shunt, as a function of coupling factor $k_{j}$. The quantity $\omega_{\mathrm{e}}^{\mathrm{PP}} / \omega_{j}$ is shown instead of $\omega_{\mathrm{e}}^{\mathrm{PP}}$. (b) Optimal value of the dimensionless electric damping $\xi_{\mathrm{e}}^{\mathrm{PP}}$ for resonant shunt, as a function of coupling factor $k_{j}$. '-', value of equation (28) with no structural damping $\left(\xi_{j}=0\right)$; ' $-\circ-$ ', numerical values $\omega_{\mathrm{e}}^{\text {PPnum }}$ and $\xi_{\mathrm{e}}^{\text {PPnum }}$ for several values of the structural damping $\xi_{j}$ (For $\xi_{j} \in\{1 \%, 0.1 \%, 0.01 \%\}$, all curves are merged).

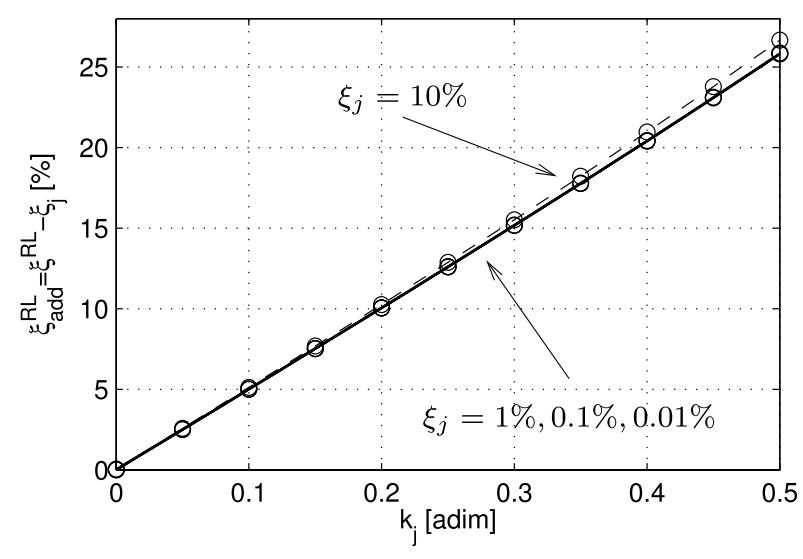

Figure 7. Added damping $\xi_{\text {add }}^{\mathrm{RL}}$ in the case of a resistive shunt, for several values of the structural damping $\xi_{j}$. '-', $\xi_{\text {add }}^{\text {RL }}$ is computed with $\left(\omega_{\mathrm{e}}, \xi_{\mathrm{e}}\right)=\left(\omega_{\mathrm{e}}^{\mathrm{PP}}, \xi_{\mathrm{e}}^{\mathrm{PP}}\right)$ (all curves are merged for $\xi_{j} \in$ $\{1 \%, 0.1 \%, 0.01 \%\})$. ' $\bigcirc$ ', $\xi_{\text {add }}^{\mathrm{RL}}$ is computed with $\left(\omega_{\mathrm{e}}, \xi_{\mathrm{e}}\right)=\left(\omega_{\mathrm{e}}^{\text {PPnum }}, \xi_{\mathrm{e}}^{\text {PPnum }}\right)$ (all points are merged).

the transfer function poles with $\left(\omega_{\mathrm{e}}, \xi_{\mathrm{e}}\right)=\left(\omega_{\mathrm{e}}^{\text {PPnum }}, \xi_{\mathrm{e}}^{\text {PPnum }}\right)$. Since all curves are almost merged into a single one, one can verify that $\left(\omega_{\mathrm{e}}^{\mathrm{PP}}, \xi_{\mathrm{e}}^{\mathrm{PP}}\right)$ remains a good estimation for the optimal electric parameter, for $\xi_{j}$ up to $10 \%$. This last result enhances the validity range, reduced to $\xi_{j}<1 \%$ at the end of section 4.1.1.

Moreover, it shows that the added damping $\xi_{\text {add }}^{\mathrm{RL}}$ does not depend on the structural damping $\xi_{j}$, so that it is perfectly known by its value with no structural damping: $\xi_{\text {add }}^{\mathrm{RL}} \simeq \xi_{0}^{\mathrm{RL}}$. As a consequence, the total damping with a resonant shunt is known analytically by the following formula (valid for $\left.\xi_{j}<10 \%\right)$ :

$$
\xi_{\mathrm{tot}}^{\mathrm{RL}}=\xi_{j}+\xi_{\mathrm{add}}^{\mathrm{RL}} \simeq \xi_{j}+\xi_{0}^{\mathrm{RL}}
$$

with $\xi_{0}^{\mathrm{R}}$ defined by equation (30).

The damping effect of the resonant shunt is shown here to be much more powerful than that of the resistive shunt. For instance, if $k_{j}=0.15, \xi_{\text {add }}^{\mathrm{RL}}=7.5 \%$ whereas $\xi_{\text {add }}^{\mathrm{R}}=0.6 \%$.
Consequently, even for high structural damping such as $\xi_{j}$ of the order of $10 \%$, the resonant shunt has a significant effect.

\section{2. $R L$-shunt forced response}

4.2.1. Shunt optimal tuning: transfer function technique. In the same way as in the resistive shunt case, the transfer function (TF) criterion is used here. The frequency response function displacement/force (under harmonic forcing of frequency $\Omega$ ), associated with equations $(27 a)$ and (27b), is written as

$$
\begin{aligned}
H(\Omega) & =\left\{1-\frac{\Omega^{2}}{\omega_{\mathrm{e}}^{2}}+2 \mathrm{j} \xi_{\mathrm{e}} \frac{\Omega}{\omega_{\mathrm{e}}}\right\} \\
& \times\left\{\frac{\Omega^{4}}{\omega_{\mathrm{e}}^{2}}-\Omega^{2}\left(1+\frac{\hat{\omega}_{j}^{2}}{\omega_{\mathrm{e}}^{2}}+4 \xi_{\mathrm{e}} \xi_{j} \frac{\omega_{j}}{\omega_{\mathrm{e}}}\right)\right. \\
& \left.+\omega_{j}^{2}+2 \mathrm{j} \Omega\left[\frac{\xi_{\mathrm{e}}}{\omega_{\mathrm{e}}}\left(\hat{\omega}_{j}^{2}-\Omega^{2}\right)+\xi_{j} \omega_{j}\left(1-\frac{\Omega^{2}}{\omega_{\mathrm{e}}^{2}}\right)\right]\right\}^{-1} .
\end{aligned}
$$

As suggested in $[5,7,40]$, the optimization problem is solved by exploiting the fact that if the structural damping $\xi_{j}$ is neglected, for a given $\omega_{\mathrm{e}}$ value, there exist two points $F^{-}$ and $F^{+}$that are common to all gain curves $|H(\Omega)|$ when $\xi_{\mathrm{e}}$ is varied (see figure 8(a)). If $\omega_{\mathrm{e}}$ is varied, the vertical positions of $F^{-}$and $F^{+}$change in an opposite way. Consequently, finding the optimum values of $\omega_{\mathrm{e}}$ and $\xi_{\mathrm{e}}$ is done in two steps. First, one has to chose $\omega_{\mathrm{e}}$ so that the vertical positions of $F^{-}$and $F^{+}$are the same (figure 8(a)). It leads to $\omega_{\mathrm{e}}=\omega_{\mathrm{e}}^{\mathrm{TF}}$ with

$$
\omega_{\mathrm{e}}^{\mathrm{TF}}=\hat{\omega}_{j}=\omega_{j} \sqrt{1+k_{j}^{2}}
$$

If $\omega_{\mathrm{e}}=\omega_{\mathrm{e}}^{\mathrm{TF}}$ and $\xi_{\mathrm{e}}$ is varied, for low values, the gain curve shows two peaks at almost the same amplitude, and for high values the two peaks collapse into only one, tending to the open circuit condition (for $\xi_{\mathrm{e}} \rightarrow+\infty$ ). The optimum choice for $\xi_{\mathrm{e}}$ would be to make both peaks coincide with 

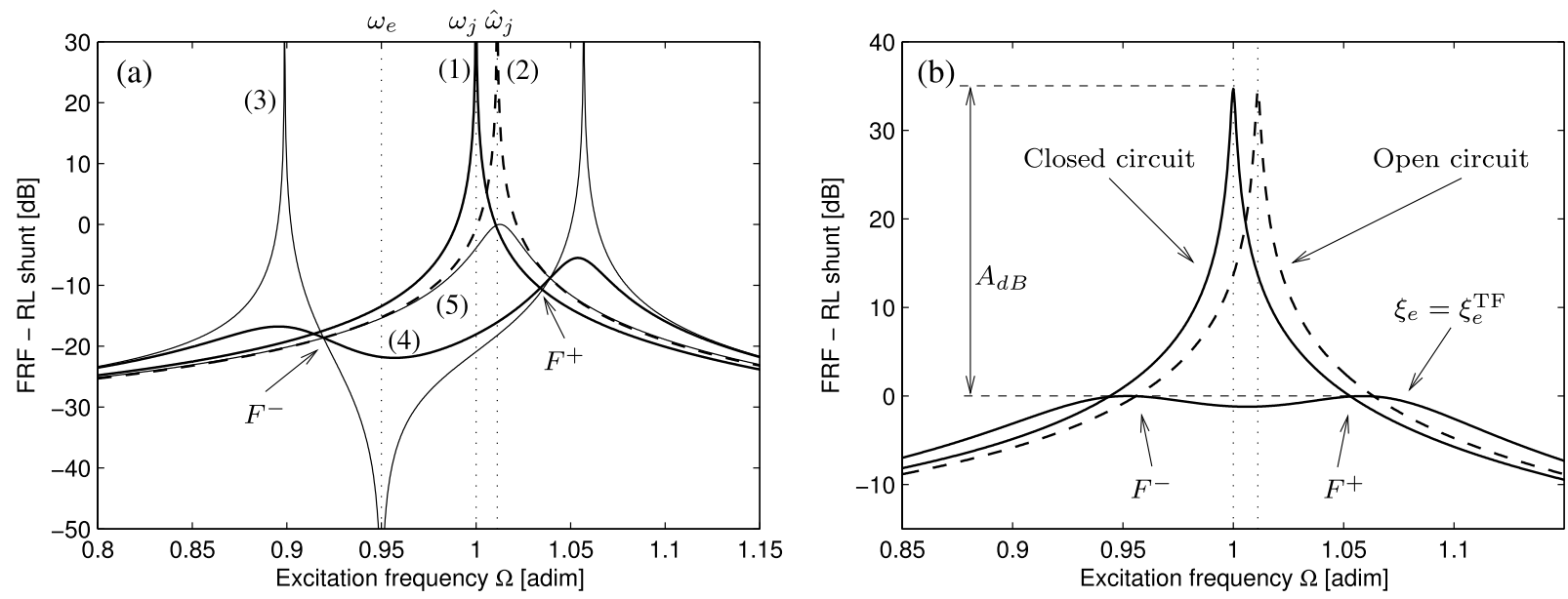

Figure 8. Frequency response function with resonant shunt for several values of the electric damping factor $\xi_{\mathrm{e}}$ (i.e. for various resistances $R)$. (a) With no structural damping $\left(\xi_{j}=0\right)$ and detuned condition $\left(\omega_{\mathrm{e}}<\omega_{\mathrm{e}}^{\mathrm{TF}}\right)$ : (1) short circuit $\left(\omega_{\mathrm{e}} \rightarrow+\infty\right)$; (2) open circuit $\left(\xi_{\mathrm{e}} \rightarrow+\infty\right)$; (3) $\xi_{\mathrm{e}}=0$; (4), (5) increasing non-zero $\xi_{\mathrm{e}}=0$. (b) With structural damping $\xi_{j}=0.1 \%$ and tuned condition $\left(\omega_{\mathrm{e}}=\omega_{\mathrm{e}}^{\mathrm{TF}}\right)$.

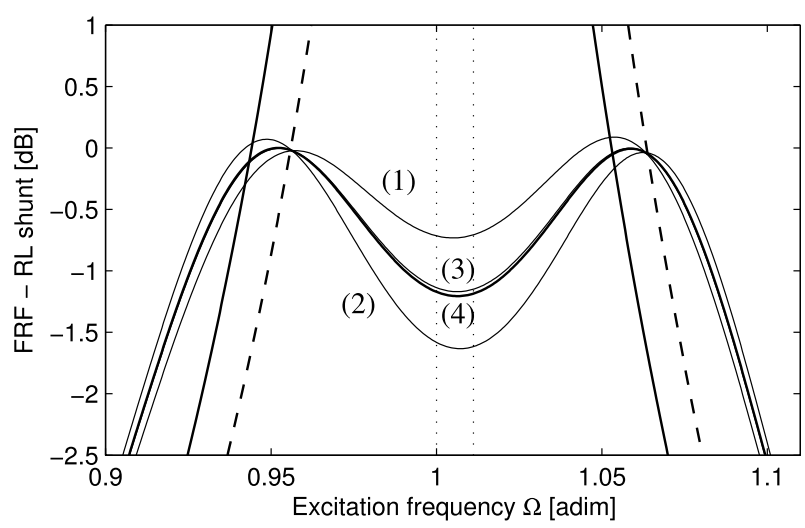

Figure 9. Frequency response function with a resonant shunt, in tuned condition $\left(\omega_{\mathrm{e}}=\omega_{\mathrm{e}}^{\mathrm{TF}}\right)$ and with structural damping $\xi_{j}=0.1 \%$, for several values of the electric damping factor $\xi_{\mathrm{e}}$ close to the optimum one: (1) $\xi_{\mathrm{e}}=\xi_{\mathrm{e}}^{-}$; (2) $\xi_{\mathrm{e}}=\xi_{\mathrm{e}}^{+} ;$(3) $\xi_{\mathrm{e}}=\left(\xi_{\mathrm{e}}^{+}+\xi_{\mathrm{e}}^{-}\right) / 2$; (4) $\xi_{\mathrm{e}}=\xi_{\mathrm{e}}^{\mathrm{TF}}$.

points $F^{-}$and $F^{+}$. This is actually impossible. However, there exist two particular values of $\xi_{\mathrm{e}}$, denoted $\xi_{\mathrm{e}}^{-}$and $\xi_{\mathrm{e}}^{+}$, so that the left peak of the gain curve coincides with $F^{-}$and the right peak with $F^{+}$, respectively. The corresponding gain curves are shown in figure 9 , along with the one associated with the mean value of $\xi_{\mathrm{e}}^{-}$and $\xi_{\mathrm{e}}^{+}$. The chosen optimal value for $\xi_{\mathrm{e}}$ is obtained by a truncated Taylor expansion of $\left(\xi_{\mathrm{e}}^{+}+\xi_{\mathrm{e}}^{-}\right) / 2$ and is (see appendix A.3)

$$
\xi_{\mathrm{e}}^{\mathrm{TF}}=\sqrt{\frac{3}{8}} k_{j} .
$$

Since the above optimum values $\left(\omega_{\mathrm{e}}^{\mathrm{TF}}, \xi_{\mathrm{e}}^{\mathrm{TF}}\right)$ are valid with no structural damping, one can numerically compute the values $\left(\omega_{\mathrm{e}}^{\text {TFnum }}, \xi_{\mathrm{e}}^{\text {TFnum }}\right)$ that minimize the peak amplitude of $|H(\Omega)|$ in the case $\xi_{j} \neq 0$. One can show that no significant difference is obtained as long as the structural damping is small (lower than 10\%).
4.2.2. Estimation of vibration reduction. To estimate the vibration reduction brought about by the resonant shunt, we use the same quantity as for the resistive shunt: the attenuation $A_{\mathrm{dB}}$ defined by equation (22) and shown in figure 8(b). With non-zero structural damping, the gain curves no longer cross at point $F^{+}$and $F^{-}$but remain very close to this condition (see figure 9) if $\xi_{j}$ is small. A good estimation of the gain curve peak amplitude with the shunt optimally tuned is then obtained by the gain at point $F^{-}: H_{\mathrm{opt}}^{\mathrm{RL}}=\left|H\left(\omega^{-}\right)\right|$ with $\omega_{\mathrm{e}}=\omega_{\mathrm{e}}^{\mathrm{TF}}$ and $\xi_{\mathrm{e}}=\xi_{\mathrm{e}}^{\mathrm{TF}}$. Using symbolic mathematical manipulations, one can show that the maximal attenuation brought by the resonant shunt can be written as

$$
\begin{aligned}
& A_{\mathrm{dB}}^{\mathrm{RL}}=20 \log \frac{H_{\mathrm{SC}}}{H_{\mathrm{opt}}} \simeq 20 \log \frac{H_{\mathrm{SC}}}{\left|H\left(\omega^{-}\right)\right|_{\omega_{\mathrm{e}}=\omega_{\mathrm{e}}^{\mathrm{TF}}, \xi_{\mathrm{e}}=\xi_{\mathrm{e}}^{\mathrm{TF}}}} \\
& =10 \log f\left(k_{j}, \xi_{j}\right),
\end{aligned}
$$

where $f\left(k_{j}, \xi_{j}\right)$ is a function of $k_{j}$ and $\xi_{j}$ only, whose expression is given by equation (A.16) in appendix A.3.

The approximation used to obtain the above analytical result is now verified, by numerically computing $H_{\mathrm{opt}}$ as the maximum value of $|H(\Omega)|$ as a function of $\Omega$, with $\left(\omega_{\mathrm{e}}, \xi_{\mathrm{e}}\right)$ adjusted to their optimal numerical values $\left(\omega_{\mathrm{e}}^{\text {TFnum }}, \xi_{\mathrm{e}}^{\text {TFnum }}\right)$. Since insignificant differences are observed for $\xi_{j}<10 \%$ (see figure 10), the vibration level attenuation brought by the resonant shunt is given by equation (35)). The a priori analogous expression for $f\left(k_{j}, \xi_{j}\right)$ obtained by considering the gain close to point $F^{+}$(i.e. with $H_{\text {opt }}=$ $\left.\left|H\left(\omega^{+}\right)\right|_{\omega_{\mathrm{e}}=\omega_{\mathrm{e}}^{\mathrm{TF}}, \xi_{\mathrm{e}}=\xi_{\mathrm{e}}^{\mathrm{TF}}}\right)$ has also been tested. It has been found that it gives a slightly different estimation of $A_{\mathrm{dB}}^{\mathrm{RL}}$, less accurate that the one of equation (35).

The above equation (35) is useful to evaluate the performance of the resonant shunt, that depends only on the modal coupling factor $k_{j}$ and the structural damping $\xi_{j}$. Figure 10 shows $A_{\mathrm{dB}}^{\mathrm{RL}}$ as a function of $k_{j}$, for several values of $\xi_{j}$. In the same way as in the case of the free response, the resonant shunt is much more powerful than the resistive shunt, even for high structural damping. For instance, if $k_{j}=0.15$, 


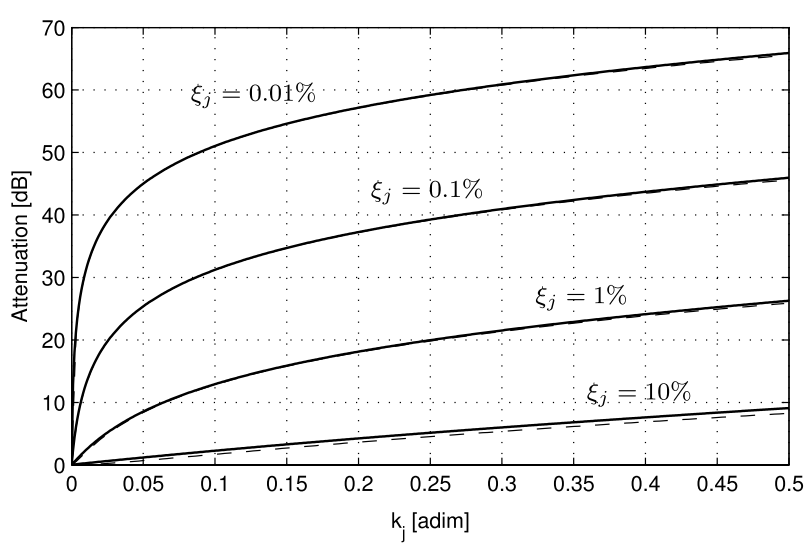

Figure 10. Attenuation $A_{\mathrm{dB}}^{\mathrm{RL}}$ brought about by the resonant shunt as a function of coupling factor $k_{j}$, for several values of structural damping $\xi_{j}$. For each value of $\xi_{j}$, two curves are almost merged: 'the one of equation (35) and '---' the one numerically computed with $\left(\xi_{\mathrm{e}}, \omega_{\mathrm{e}}\right)=\left(\xi_{\mathrm{e}}^{\mathrm{TFnum}}, \omega_{\mathrm{e}}^{\mathrm{TFnum}}\right)$.

up to $55 \mathrm{~dB}$ of attenuation are obtained with $\xi_{j}=0.01 \%$ of structural damping and $3 \mathrm{~dB}$ are observed with $\xi_{j}=10 \%$. As for $A_{\mathrm{dB}}^{\mathrm{R}}, A_{\mathrm{dB}}^{\mathrm{RL}}$ does not tends to zero when $k_{j}$ tends to zero, for the same reason.

\section{Experiments}

In order to validate some of the theoretical results of this paper, experiments have been performed on the beam sketched in figure 11. Some of the present experimental results have been used to validate a finite-element model in [39] and an analytical model in [35]. The beam is made of aluminum and the piezoelectric material is PIC151. The beam and patch lengths are $l_{b}=170 \mathrm{~mm}$ and $l_{p}=25 \mathrm{~mm}$, their thicknesses are $h_{b}=2 \mathrm{~mm}$ and $h_{b}=0.5 \mathrm{~mm}$, their common width is $b=20 \mathrm{~mm}$ and the patch position is $x_{-}=0.5 \mathrm{~mm}$. The patch length has been chosen first and then, knowing $l_{p} / l_{b}=0.147$, the patch thickness has been selected as close as possible to the optimal one found in [35], to maximize the piezoelectric coupling $k_{1}$ of the first mode.

The two piezoelectric patches are carefully bonded on the beam with an Araldite 2011 epoxy adhesive [41]. The whole bonding procedure, based on the one used for a strain gauge [42], is fully detailed in [43]. The obtained epoxy layer thickness has been measured to about $0.02 \mathrm{~mm}$, which justifies the perfect bonding hypothesis of the model. The piezoelectric patches have wrapped electrodes and are installed on the beam with opposite poling directions. They are connected in series to the shunt circuit. The shunt circuit can be either a simple resistor (practically realized with potentiometers) for the resistive shunt, or a resistor in series with an inductor for the resonant shunt (figure 1). In the latter case, the practical values for the inductance $L$ necessary to tune the RL-shunt on one mechanical resonance (of the order of $100 \mathrm{H}$ for the first beam modes) are too large to be realized with a passive electronic component. A synthetic inductor, realized with operational amplifiers, is here used. The practical details are gathered in appendix B.

The beam is clamped with a laboratory made vice, with one of its chops articulated with respect to the other to obtain a boundary condition as perfect as possible (figure 11). An non-contact electromagnetic driving system is used, composed of a small magnet glued on the structure with bees' wax, subjected to the magnetic field created by a coil, fed by a broadband noise electrical signal. This exciter is fully described in [44]. The force acting on the beam is estimated by measuring the current intensity in the coil, proportional to the force. Current monitoring available on the power amplifier is used for this. The beam motion is obtained with a laser Doppler vibrometer, that measures the velocity of the beam at one point.

\subsection{Shunt performance in forced vibrations}

The beam is studied under six electromechanical conditions, enumerated below:

- (SC): the shunt is short-circuited with a wire (it imposes $V=0$ on figure 1$)$;

- (OC): the shunt is left open (it imposes $I=\dot{Q}=0$ on figure 1);

- (R1): a resistive shunt, tuned to the first beam mode (1F), is connected to the piezoelectric patches;

- (R2): a resistive shunt, tuned to the second beam mode $(2 \mathrm{~F})$, is connected to the piezoelectric patches;

- (RL1): a resonant shunt, tuned to the first beam mode (1F), is connected to the piezoelectric patches;

- (RL2): a resonant shunt, tuned to the second beam mode $(2 \mathrm{~F})$, is connected to the piezoelectric patches.
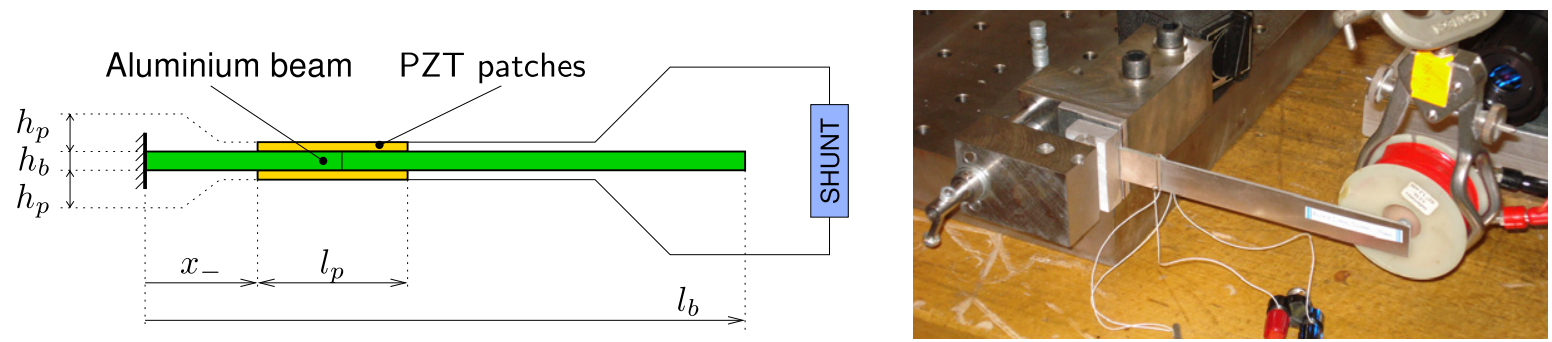

Figure 11. Sketch and photograph of the experimental setup. 

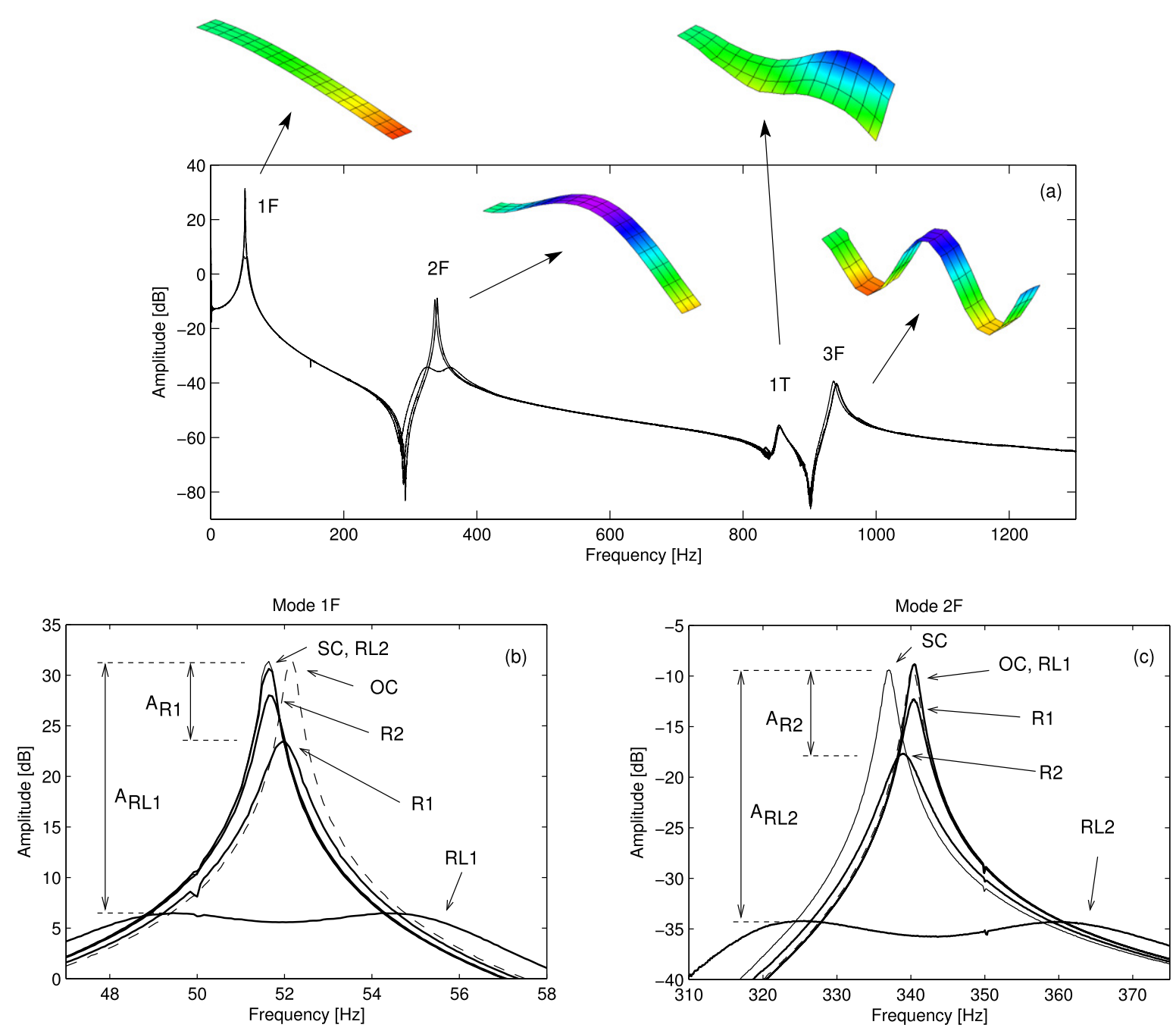

Figure 12. Experimental frequency response function of the beam of figure 11, measured near the beam free end, for six electromechanical conditions: (SC), short circuit; (OC), open circuit; $\left(R_{1}\right),\left(R L_{1}\right)$, resistive and resonant shunt tuned to mode $1 F$; $\left(R_{2}\right),\left(R L_{2}\right)$, resistive and resonant shunt tuned to mode $2 \mathrm{~F}$. (a) General view showing the first four beam resonances and mode shapes, measured by a scanning laser vibrometer. (b) Detail of mode $1 \mathrm{~F}$ resonance. (c) Detail of mode $2 \mathrm{~F}$ resonance.

In each condition, the beam's frequency response function, between the point forcing and the tip displacement, is measured and shown in figure 12.

The natural frequencies of the beam, in short circuit (SC) as well as in open circuit (OC) conditions, are first estimated by measuring the frequency peaks of the corresponding FRFs (figure 12). Since the damping is very low and because a high frequency resolution $(78 \mathrm{mHz})$ is used for the measured FRFs, the resonance peaks are very sharp and the resonance frequencies give a good estimation of the natural frequencies. The first four natural frequencies (corresponding to three flexural modes $1 \mathrm{~F}, 2 \mathrm{~F}$ and $3 \mathrm{~F}$, and one torsional mode $1 \mathrm{~T}$ ) in both conditions (denoted as $f_{j}$ in SC and $\hat{f}_{i}$ in OC) are given in table 1 . The damping factor of the first two modes is measured by the frequency bandwidth $\Delta \omega$ at $-3 \mathrm{~dB}$ below the short circuit resonance peak, with the formula $\xi_{j}=\Delta \omega_{j} /\left(2 \omega_{j}\right)$. An

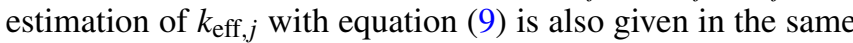
table, which is of the order of $14 \%$ for the first two modes. The $1 \mathrm{~T}$ torsional mode has a low coupling coefficient compared to those of the flexural modes. It would be zero in theory, since the extensional motion of the piezoelectric patches are not activated when the beam undergoes torsion.

Now, the vibration response of the beam connected to the four shunt circuits is analyzed. Theoretical values of the optimal resistance and inductance are indicated in table 1. They are computed using the formula gathered in table 3 , with the experimental short circuit frequencies $f_{j}=\omega_{j} /(2 \pi)$ and the equivalent theoretical capacitance of the two piezoelectric patches connected in series $C=C_{0} / 2=9.16 \mathrm{nF}$, with the capacitance of each patch being $C_{0}=\epsilon_{33} b l_{p} / h_{p}$, with $\epsilon_{33}=$ $2068 \epsilon_{0}$ the permittivity of PIC $151[39,45]$. The high values for the inductances justify the use of the synthetic inductor. In both conditions, R-shunt and RL-shunt, the tuning was carefully adjusted manually to obtain the highest attenuation. The final experimental values of the electrical parameters have not been measured, but they are close to the theoretical ones. The experimental attenuations (in decibels) achieved by the shunts are gathered in table 1 and are shown in figures 12(b) and (c). Since the structure is lightly damped, the vibration reduction achieved by the shunts is excellent: the resistive 

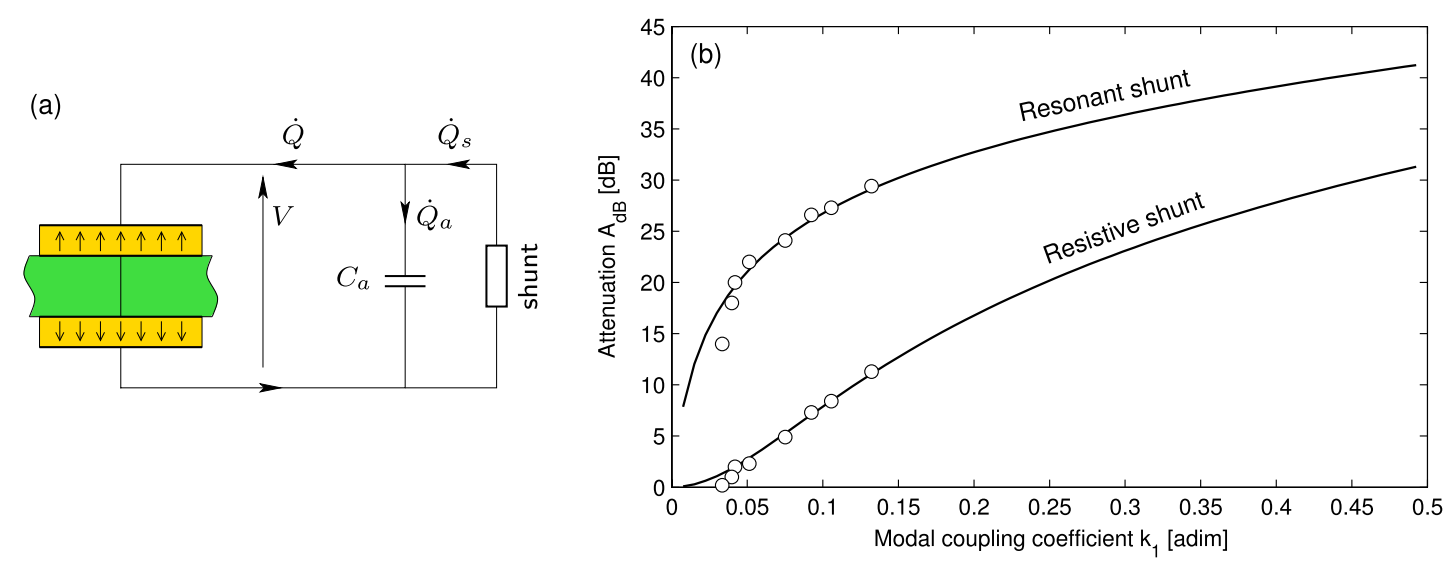

Figure 13. (a) Shunt circuit connected to the piezoelectric patches with an added capacitance $C_{\mathrm{a}}$ in parallel. (b) Attenuation $A_{\mathrm{dB}}$ achieved by resistive and resonant shunts as a function of coupling factor $k_{1}$ of mode $1 \mathrm{~F}$, for the measured damping $\xi_{1}=0.17 \%$. '-', theoretical results from equations (24) and (35); 'o', experimental results obtained by artificially increasing the electric capacitance of the piezoelectric patches.

Table 1. Experimental results in forced vibration (Exp., experimental; Th., theoretical).

\begin{tabular}{|c|c|c|c|c|c|c|c|}
\hline & & & & Mode 1F & Mode $2 \mathrm{~F}$ & Mode 1T & Mode $3 F$ \\
\hline \multirow[t]{2}{*}{ Frequencies } & Short circuit (SC) $f_{j}$ & Exp. & $(\mathrm{Hz})$ & 51.64 & 337.0 & 853.0 & 936.3 \\
\hline & Open circuit (OC) $\hat{f}_{j}$ & Exp. & $(\mathrm{Hz})$ & 52.17 & 340.2 & 854 & 940.0 \\
\hline Structural damping factors & $\xi_{j}$ & Exp. & $(\%)$ & 0.34 & 0.28 & - & - \\
\hline Effective coupling factors & $k_{\mathrm{eff}, j}$ & Exp. & $(\operatorname{adim})$ & 0.144 & 0.138 & 0.050 & 0.089 \\
\hline \multirow[t]{3}{*}{ Optimal parameters } & R-shunt resistance & Th. & $(\mathrm{k} \Omega)$ & 335 & 51 & - & - \\
\hline & RL-shunt inductance & Th. & $(\mathrm{H})$ & 1020 & 23.9 & - & - \\
\hline & RL-shunt resistance & Th. & $(\mathrm{k} \Omega)$ & 58.62 & 8.65 & - & - \\
\hline \multirow[t]{8}{*}{ Attenuations $A_{\mathrm{dB}}$} & R1-shunt & Exp. & $(\mathrm{dB})$ & 7.9 & 2.9 & $<0.5$ & 1.5 \\
\hline & & Th. & $(\mathrm{dB})$ & 8.1 & - & - & - \\
\hline & R2-shunt & Exp. & $(\mathrm{dB})$ & 3.4 & 8.3 & $<0.5$ & 3.5 \\
\hline & & Th. & $(\mathrm{dB})$ & - & 8.6 & & \\
\hline & RL1-shunt & Exp. & $(\mathrm{dB})$ & 24.9 & 0 & $<0.5$ & 0.5 \\
\hline & & Th. & $(\mathrm{dB})$ & 24.1 & - & - & - \\
\hline & RL2-shunt & Exp. & $(\mathrm{dB})$ & 0.7 & 24.9 & $<0.5$ & 1 \\
\hline & & Th. & $(\mathrm{dB})$ & - & 25.7 & - & - \\
\hline
\end{tabular}

shunts reduce the first two resonance peaks by about $8 \mathrm{~dB}$ whereas the resonant shunts reduce them by $25 \mathrm{~dB}$. The selectivity of the two shunt techniques is also visible: the resistive shunt has a more broadband effect than the resonant shunt. The R-shunt tuned on mode $1 \mathrm{~F}$ significantly reduces the $2 \mathrm{~F}$ response $(3 \mathrm{~dB})$ and vice versa, whereas the resonant shunt effect on other modes is negligible.

The theoretical values of the attenuation achieved by the shunt are also given in table 1 . They have been computed with equations (24) and (35), using the measured values of $k_{\mathrm{eff}, j} \simeq$ $k_{j}$ and $\xi_{j}$. Considering that the experimental determination of $k_{\text {eff, } j}$ and $\xi_{j}$ is associated with poor confidence, the agreement between experiments and theory for $A_{\mathrm{dB}}$ is excellent, thus validating the closed-form expressions obtained in this paper.

\subsection{Electromechanical coupling variation}

In this section, an experimental validation of the theoretical vibration reduction $A_{\mathrm{dB}}$ obtained in sections 3.2.2 and 4.2.2 is proposed, by artificially reducing the electromechanical coupling to measure several points on figures 5 and 10 . For this, a known capacitance is added in parallel to the shunt (figure 13(a)). The equivalent capacitance of the piezoelectric patches is then artificially increased, which leads to a decrease in the electromechanical coupling factor. This feature has been observed in [10]. Moreover, this technique is also used in the opposite way with a negative (synthetic and active) capacitance, to increase the coupling factor and improve the shunt performance [12].

Quantitatively, if a capacitance $C_{\mathrm{a}}$ is added in parallel to the electrical circuit (figure 13(a)), the following equations hold:

$$
V=\frac{Q_{\mathrm{a}}}{C_{\mathrm{a}}}, \quad Q=Q_{\mathrm{s}}-Q_{\mathrm{a}},
$$

where $Q_{\mathrm{a}}$ and $Q_{\mathrm{s}}$ are the charges that flow respectively through $C_{\mathrm{a}}$ and the shunt. Equation $(2 b)$ can be replaced by

$$
\left(C+C_{\mathrm{a}}\right) V-Q_{\mathrm{s}}+\sum_{i=1}^{N} \chi_{i} q_{i}=0
$$



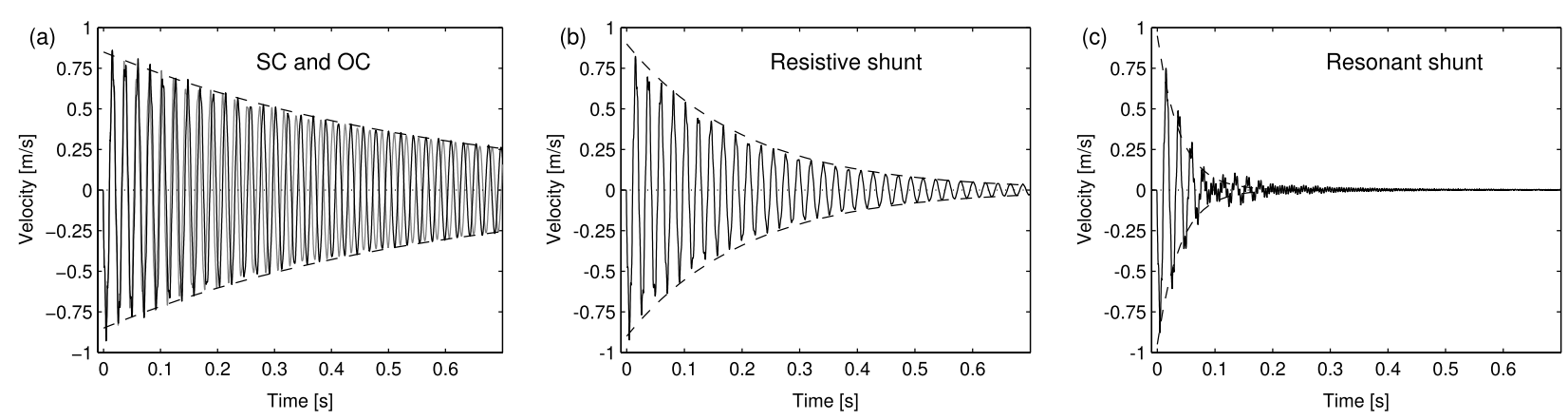

Figure 14. Time evolution of the beam tip velocity after initial static deflection and zero initial velocity: (a) short circuit (black) and open circuit conditions (gray); (b) with resistive shunt tuned on mode 1F; (c) with resonant shunt tuned on mode 1F. '- - -', exponential envelopes.

so that $C+C_{\mathrm{a}}$ is now the equivalent capacitance viewed by the shunt. It thus leads to the replacement of $C$ by $C+C_{\mathrm{a}}$ in the reduced parameter definitions (4), so that $k_{j}$ in equation (6) is replaced by the reduced coupling factor:

$$
\tilde{k}_{j}=\frac{k_{j}}{\beta}, \quad \text { with } \beta=\sqrt{1+\frac{C_{\mathrm{a}}}{C}},
$$

which shows the reduction effect of a positive added capacitance $C_{\mathrm{a}}$. All equations have the same form, with $k_{j}$ replaced by $\tilde{k}_{j}, \bar{Q}$ replaced by $\bar{Q}_{\mathrm{s}} / \beta$ and $V$ replaced by $\beta \bar{V}$. The mechanical part is consequently unchanged.

The first $(1 \mathrm{~F})$ mode of the beam is considered and increasing capacitances $C_{\mathrm{a}}$ are added in parallel to the piezoelectric patches. For each capacitance, the effective coupling factor $k_{\text {eff, } 1}$ of the first mode is estimated by measuring the open circuit and short circuit natural frequencies. In fact, only the open circuit natural frequency changes with the added capacitance. The structural damping $\xi_{1}$ is also measured with the frequency bandwidth method. Its value is $\xi_{1}=0.17 \%$, different from the value measured for the experiments of section 5.1, probably because of the clamped boundary, since the beam has been unmounted between both experiments. Then, both resistive and resonant shunts are tuned on the first mode and the corresponding attenuation $A_{\mathrm{dB}}$ is measured. Then, these experimental gain reductions are compared with the theoretical ones, calculated by equations (24) and (35) with the above measured values of $k_{1} \simeq k_{\mathrm{eff}, 1}$ and $\xi_{1}$, on figure $13(\mathrm{~b})$. It shows an excellent agreement and thus validates the theoretical results of sections 3.2.2 and 4.2.2. Below coupling factors of $4 \%$, it has been found difficult to properly tune the resonant shunt.

\subsection{An example in free vibrations}

Some experimental results in free vibrations are now described. The beam is subjected to an initial static deformed position, created by a constant electric current fed to the coil. At the initial time, the electric current is reduced to zero (by opening a switch) and a very reproducible free vibration regime is obtained. It is recorded in the four following conditions: with the piezoelectric patches in short circuit (SC), in open circuit (OC) and connected to resistive and resonant shunt tuned to the first beam resonance with the transfer function criterion ( $\mathrm{R}$ and $\mathrm{RL}$ ). The two latter conditions have the same tuning as those used for the forced response of section 5.1. The obtained time evolutions are shown in figure 14. Since the constant force that imposes the static initial position is localized near the tip of the beam, the associated deflection is very close to the first (1F) beam mode shape. As a consequence, the two shunts, tuned on the $1 \mathrm{~F}$ mode, have a large damping effect on the oscillations, clearly visible on figure 14 . In the case of the resonant shunt, a slight beating phenomenon is visible, probably stemming from a slight mistuning of the electrical frequency (corresponding to the inductance), so that the two poles of the transfer function do not have the same frequencies (their imaginary part), which is the optimal condition of the PP criterion (section 3.1.1). This mistuning can be either due to an experimental uncertainty or to the slight difference in tuning condition between the transfer function criterion and the PP criterion (see table 3). High frequency oscillations, not damped by the resonant shunt and related to the higher modes, are also observable at the end of the signal. Finally, another interesting feature is that the slight difference between the open circuit and the short circuit natural frequencies is clearly visible on figure 14(a), since the pseudo-periods of the two oscillations are slightly different, thus leading to a progressive phase shift.

In each of the four conditions (SC, OC, R and RL), a decaying exponential can be adjusted to fit the envelopes of the free vibration curves, in order to estimate equivalent damping factors. Those exponential envelopes are shown in figure 14 and the corresponding damping factors are collected in table 2 (third row). By subtracting the experimental damping factor in both shunt conditions from its value in short circuit, added damping factors $\xi_{\text {add }}$ are obtained (fourth row) and can be compared to their theoretical values (fifth row), estimated with equation (16) for the resistive shunt and equation (30) for the resonant shunt, that depends only on the electromechanical coupling factor. The latter (second row) is here estimated with equation (9), where the natural frequencies in short and open circuit $f_{1}^{\mathrm{SC}}$ and $f_{1}^{\mathrm{OC}}$ (first row) are the average periods of oscillations of the system in SC and OC conditions, estimated with the curves of figure 14(a). 
Table 2. Experimental results in free vibration (Exp., experimental; Th., theoretical).

\begin{tabular}{lllllll}
\hline & & & SC & OC & R & RL \\
\hline First mode natural frequencies $f_{1}^{\mathrm{SC}}, f_{1}^{\mathrm{OC}}$ & $(\mathrm{Hz})$ & Exp. & 45.55 & 46.58 & - & - \\
EEMCF $k_{\text {eff, }}$ & $(\%)$ & Exp. & & 21.5 & - & - \\
Damping factors $\xi$ & $(\%)$ & Exp. & 0.6 & - & 1.7 & 9 \\
Added damping factors $\xi_{\text {add }}=\xi^{\text {shunt }}-\xi^{\mathrm{SC}}$ & $(\%)$ & Exp. & - & - & $\mathbf{1 . 1}$ & $\mathbf{8 . 4}$ \\
Added damping factors $\xi_{\text {add }}=\xi^{\text {shunt }}-\xi^{\mathrm{SC}}$ & $(\%)$ & Th. & - & - & $\mathbf{1 . 1 5}$ & $\mathbf{1 0 . 7}$ \\
\hline
\end{tabular}

Table 3. Optimal electrical parameter values for the tuned resistive and resonant shunt and associated performances. All parameters are dimensioned. $\omega_{j}$ is the $j$ th structure's natural angular frequency with the shunt short-circuited (in $\left.\left(\mathrm{rad} \mathrm{s}^{-1}\right)\right),\left(k_{j}, \xi_{j}\right)$ are the dimensionless $j$ th modal coupling factor and damping factor and $C$ is the equivalent piezoelectric patch capacitance (in (F)). The $R$ and $L$ values are obtained by combining equations (11), (13) and (21) for the resistive shunt, and equations (26), (28), (33) and (34) for the resonant shunt.

\begin{tabular}{llll}
\hline & & $\begin{array}{l}\text { Free response } \\
\text { Pole placement criterion }\end{array}$ & $\begin{array}{l}\text { Forced response } \\
\text { Transfer function criterion }\end{array}$ \\
\hline Resistive shunt & Opt. resistance & $R=\frac{1}{C \omega_{j}\left(1+k_{j}^{2} / 2\right)}$ & $R=\frac{1}{C \omega_{j} \sqrt{1+k_{j}^{2} / 2}}$ \\
& Performances & $\xi_{\mathrm{add}}^{\mathrm{R}}=\frac{k_{j}^{2}}{4 \sqrt{1+k_{j}^{2} / 2-k_{j}^{4} / 16} \simeq \frac{k_{j}^{2}}{4}}$ & $A_{\mathrm{dB}}^{\mathrm{R}}=20 \log \frac{k_{j}^{2}+2 \sqrt{2} \xi_{j} \sqrt{2+k_{j}^{2}}}{4 \xi_{j} \sqrt{1-\xi_{j}^{2}}}$ \\
Resonant shunt & Opt. resistance & $R=\frac{2 k_{j}}{C \omega_{j}\left(1+k_{j}^{2}\right)^{3 / 2}}$ & $R=\sqrt{\frac{3}{2}} \frac{k_{j}}{C \omega_{j} \sqrt{1+k_{j}^{2}}}$ \\
& Opt. inductance & $L=\frac{1}{C \omega_{j}^{2}\left(1+k_{j}^{2}\right)^{2}}$ & $L=\frac{1}{C \omega_{j}^{2}\left(1+k_{j}^{2}\right)}$ \\
& Performances & $\xi_{\mathrm{add}}^{\mathrm{RL}}=\frac{\left|k_{j}\right|}{\sqrt{4-k_{j}^{2}}} \simeq \frac{\left|k_{j}\right|}{2}$ & $\begin{array}{l}A_{\mathrm{dB}}^{\mathrm{RL}}=A_{\mathrm{dB}}^{\mathrm{RL}}\left(k_{j}, \xi_{j}\right), \\
\text { equations }(35) \text { and (A.16) }\end{array}$ \\
\hline
\end{tabular}

In fact, a different beam than the one of sections 5.1 and 5.2, with longer piezoelectric patches, has been used in the present experiments. This explains why its characteristics are different from those of table 1: the frequencies are smaller, the MEMCF is larger $(21.5 \%)$ and it is more damped $(\xi=0.6 \%$ in SC/OC). However, an excellent match between experimental and theoretical values for the added damping is obtained. This further validates the closed-form expressions presented in this paper.

\section{Conclusion}

In this paper we first addressed the tuning of both resistive and resonant shunts. The optimal values for the electrical parameters of both R- and RL-shunts, in both free and forced vibration conditions, have been obtained, revisiting some results of the literature obtained in the case of no structural damping. Those classical results have been extended with some numerical validations, showing that they are still valid for damping factors up to $10 \%$. Those parameters are gathered in table 3. They depend only on: (i) the natural frequency in short circuit of the considered vibration mode as well as its modal coupling factor, and (ii) the equivalent electrical blocked capacity of the patches. The tuned values of the electrical parameters differ, depending on the optimization criterion used (PP or transfer function). However, for small values of the coupling factor $k_{j}$, both criteria lead to almost the same values, except for the optimal resistance of the RL-shunt, which differs by a factor of about $2 / \sqrt{3 / 2} \simeq$ 1.6. An analogous result has been obtained in the cases of synchronous switch systems [23].
Then, performance indicators were derived for both R- and RL-shunts. An added damping factor $\xi_{\text {add }}$ and an attenuation level $A_{\mathrm{dB}}$ were defined for, respectively, the free and forced vibration cases. Closed-form expressions have been obtained and are recalled in table 3. In all cases, the performances are only functions of the piezoelectric coupling factor $k_{j}$ and the structural damping factor $\xi_{j}$ of the $j$ th targeted vibration mode. As a consequence, the present results can be used for any mechanical host structure provided its modal coupling factor and the modal damping is known. Since $k_{j}$ is very close to the effective coupling factor $k_{\text {eff }}$, that depends on the natural frequencies in open and short circuit (equation (9)), the shunt performances are thus very easy to estimate with figures 3, 5, 7 and 10 and table 3. Those results are fully validated in section 5 and in figure 13 with original experiments, obtained by artificially degrading the electromechanical coupling by adding a capacitance in the shunt.

A conclusion is that the shunt performances decrease drastically when the structural damping increases. However, even if the RL-shunt has the best performance, the R-shunt is indeed efficient for very low structural damping factors. As a consequence, this technique, often considered less in the literature due to its poor performance, is probably an interesting alternative to the RL-shunt in the case of mechanical structures with very low structural damping [32]. It has the advantages of being very simple to implement, of requiring no synthetic electronic components and of having a tuning not as demanding as that of the RL-shunt. However, it can work only if the coupling factor $k_{j}$ is finely optimized, 
by carefully choosing its position, geometry and thickness, as proposed in [35, 32].

\section{Appendix A. Electrical optimization details}

\section{A.1. Resistive shunt, pole placement criterion}

We denote by $\boldsymbol{A}^{\mathrm{R}}$ the coefficient matrix of equations (12a) and (12b). The transfer function poles $\lambda_{i}$ thus verifies

$$
\begin{array}{r}
\operatorname{det} \boldsymbol{A}^{\mathrm{R}}=\tau_{\mathrm{e}} \lambda^{3}+\left(2 \xi_{j} \omega_{j} \tau_{\mathrm{e}}+1\right) \lambda^{2} \\
+\left(\tau_{\mathrm{e}} \hat{\omega}_{j}^{2}+2 \xi_{j} \omega_{j}\right) \lambda+\omega_{j}^{2}=0
\end{array}
$$

that can be rewritten as

$$
\begin{gathered}
\operatorname{det} \boldsymbol{A}^{\mathrm{R}}=\sum_{i=1}^{3} \tau_{\mathrm{e}}\left(\lambda-\lambda_{i}\right)=\tau_{\mathrm{e}}^{3} \lambda^{3}+\tau_{\mathrm{e}}(2 \mu+\sigma) \lambda^{2} \\
+\tau_{\mathrm{e}}\left(\mu^{2}+\omega^{2}+2 \mu \sigma\right) \lambda+\tau_{\mathrm{e}} \sigma\left(\mu^{2}+\omega^{2}\right) .
\end{gathered}
$$

Then, identifying the two above equations terms by terms and imposing $\xi_{j}=0$ leads to

$$
\begin{gathered}
\tau_{\mathrm{e}}(2 \mu+\sigma)=1, \quad\left(\mu^{2}+\omega^{2}+2 \mu \sigma\right)=\hat{\omega}_{j}^{2}, \\
\tau_{\mathrm{e}} \sigma\left(\mu^{2}+\omega^{2}\right)=\omega_{j}^{2} .
\end{gathered}
$$

Eliminating $\omega$ and $\sigma$ in those three equations leads to

$$
8 \tau_{\mathrm{e}} \mu^{3}-8 \mu^{2}+\left(2 \tau_{\mathrm{e}} \hat{\omega}_{j}^{2}+2 / \tau_{\mathrm{e}}\right) \mu-\omega_{j}^{2} k_{j}^{2}=0 .
$$

Derivating equation (A.4) with respect to $\tau_{\mathrm{e}}$ shows that

$$
\frac{\partial \mu}{\partial \tau_{\mathrm{e}}}=0 \Longrightarrow 8 \mu^{2}+2 \hat{\omega}_{j}^{2}-2 / \tau_{\mathrm{e}}^{2}=0 .
$$

Finally, eliminating $\mu$ between equations (A.5) and (A.4) gives the value $\tau_{\mathrm{e}}^{\mathrm{PP}}$ of $\tau_{\mathrm{e}}$ and the corresponding values for $\omega$, $\mu$ and $\sigma$ (equations (13) and (14)).

\section{A.2. Resonant shunt, pole placement criterion}

We denote by $\boldsymbol{A}^{\mathrm{RL}}$ the coefficient matrix of equations (27a) and $(27 b)$. The transfer function pole $\lambda_{i}$ thus verifies

$$
\begin{aligned}
\operatorname{det} A^{\mathrm{RL}} & =\frac{\lambda^{4}}{\omega_{\mathrm{e}}^{2}}+\frac{2}{\omega_{\mathrm{e}}}\left(\xi_{\mathrm{e}}+\xi_{j} \frac{\omega_{j}}{\omega_{\mathrm{e}}}\right) \lambda^{3} \\
+ & \left(\frac{\hat{\omega}_{j}^{2}}{\omega_{\mathrm{e}}^{2}}+4 \xi_{\mathrm{e}} \xi_{j} \frac{\omega_{j}}{\omega_{\mathrm{e}}}+1\right) \lambda^{2} \\
+ & 2\left(\frac{\xi_{\mathrm{e}}}{\omega_{\mathrm{e}}} \hat{\omega}_{j}^{2}+\xi_{j} \omega_{j}\right) \lambda+\omega_{j}^{2}=0 .
\end{aligned}
$$

that can be rewritten as

$$
\begin{aligned}
& \operatorname{det} A^{\mathrm{RL}}=\sum_{i=1}^{4} \frac{1}{\omega_{\mathrm{e}}}\left(\lambda-\lambda_{i}\right) \\
& =\frac{1}{\omega_{\mathrm{e}}}(\lambda+\mu-\mathrm{j} \omega)^{2}(\lambda+\mu+\mathrm{j} \omega)^{2} .
\end{aligned}
$$

Then, expanding and identifying the above expression with equation (A.6) and imposing $\xi_{j}=0$ leads to

$$
\begin{aligned}
\frac{2 \xi_{\mathrm{e}}}{\omega_{\mathrm{e}}} & =\frac{4 \mu}{\omega_{\mathrm{e}}^{2}}, \quad 1+\frac{\hat{\omega}_{j}^{2}}{\omega_{\mathrm{e}}^{2}}=\frac{2}{\omega_{\mathrm{e}}^{2}}\left(2 \mu^{2}+\omega^{2}\right), \\
\frac{2 \xi_{\mathrm{e}} \hat{\omega}_{j}^{2}}{\omega_{\mathrm{e}}} & =\frac{4 \mu}{\omega_{\mathrm{e}}^{2}}\left(\mu^{2}+\omega^{2}\right), \quad \omega_{j}^{2}=\frac{\left(\mu^{2}+\omega^{2}\right)^{2}}{\omega_{\mathrm{e}}^{2}} .
\end{aligned}
$$

After a few mathematical operations to solve those four equations for the four unknowns $\omega_{\mathrm{e}}, \xi_{\mathrm{e}}, \mu$ and $\omega$, equations (28) and (29) are obtained. Those results have been previously obtained by $[5,8]$ with different notation.

\section{A.3. Resonant shunt, transfer function criterion}

The first step is to write the modulus of $H(\Omega)$ (equation (18)) with $\xi_{j}=0$ in the following form:

$$
|H(\Omega)|^{2}=\frac{A_{0}+A_{2} \xi_{\mathrm{e}}^{2}}{B_{0}+B_{2} \xi_{\mathrm{e}}^{2}}
$$

with

$$
\begin{gathered}
A_{0}=\left(1-\frac{\Omega^{2}}{\omega_{\mathrm{e}}^{2}}\right)^{2}, \quad A_{2}=4 \frac{\Omega^{2}}{\omega_{\mathrm{e}}^{2}}, \\
B_{0}=\left[\frac{\Omega^{4}}{\omega_{\mathrm{e}}^{2}}-\Omega^{2}\left(1+\frac{\hat{\omega}_{j}^{2}}{\omega_{\mathrm{e}}^{2}}\right)+\omega_{j}^{2}\right]^{2}, \\
B_{2}=\frac{4 \Omega^{2}}{\omega_{\mathrm{e}}^{2}}\left(\hat{\omega}_{j}^{2}-\Omega^{2}\right) .
\end{gathered}
$$

Then, the frequencies $\omega_{-}$and $\omega_{+}$of points $F_{-}$and $F_{+}$verify

$$
\begin{aligned}
& |H(\omega)|_{\xi_{\mathrm{e}}=0}=|H(\omega)|_{\xi_{\mathrm{e}} \rightarrow+\infty}, \\
& \quad \Rightarrow A_{0} B_{2}=A_{2} B_{0}, \\
& \quad \Rightarrow \omega_{ \pm}^{2}=\frac{\hat{\omega}_{j}^{2}}{2}\left(1+\frac{\omega_{\mathrm{e}}^{2}}{\hat{\omega}_{j}^{2}} \pm \sqrt{1+\frac{\omega_{\mathrm{e}}^{4}}{\hat{\omega}_{j}^{4}}-\frac{2}{1+k_{j}^{2}}}\right) .
\end{aligned}
$$

The gain in points $F_{-}$and $F_{+}$is obtained with equation (A.12) and $\xi_{\mathrm{e}} \rightarrow+\infty$ :

$$
|H(\Omega)|^{2}=\frac{A_{2}}{B_{2}}=\frac{1}{\left(\hat{\omega}_{j}^{2}-\Omega^{2}\right)^{2}} .
$$

Then, the optimal value $\omega_{\mathrm{e}}^{\mathrm{TF}}=\hat{\omega}_{j}$ (equation (33)) is obtained by imposing $\left|H\left(\omega^{+}\right)\right|^{2}=\left|H\left(\omega^{-}\right)\right|^{2}$. Their common value is

$$
\left|H\left(\omega_{ \pm}\right)\right|_{\xi_{j}=0, \omega_{\mathrm{e}}=\omega_{\mathrm{e}}^{\mathrm{TF}}}=\sqrt{2} \sqrt{1+k_{j}^{2}} / \hat{\omega}_{j}^{2} / k_{j} .
$$

The values $\xi_{\mathrm{e}}^{ \pm}$for which the gain curve has one peak at point $F^{ \pm}$are obtained following the method introduced in [46] and fully explained in [47], for tuned mass dampers (TMD). It must be emphasized that the present RL-shunt coupled to a one degree of freedom mechanical oscillator is the analog of the TMD presented in [47], with the absorber damper connected to the ground. However, an exact analogy is not possible since in the classical TMD, the important parameter to optimize is the mass ratio $m_{\mathrm{a}} / m_{\mathrm{p}}$ (of the absorber and the 
primary system, using the notations of [47]), whereas in the RL-shunt it is the coupling factor $k_{j}$, equivalent to the stiffness ratio $k_{\mathrm{a}} / k_{\mathrm{p}}$ of the TMD. As a consequence, the results of [47] cannot be used here as they are. So, following the method of $[46,47]$, one obtains

$$
\begin{aligned}
\xi_{\mathrm{e}}^{ \pm} & =\sqrt{\frac{3}{8}} k_{j} \frac{\sqrt{2}}{\sqrt{\sqrt{1+k j^{2}}\left( \pm \sqrt{2} k_{j}+2 \sqrt{1+k_{j}^{2}}\right)}} \\
& \simeq \sqrt{\frac{3}{8}} k_{j} \frac{\sqrt{2}}{\sqrt{2 \pm \sqrt{2} k_{j}}} .
\end{aligned}
$$

whose mean value is close to $\xi_{\mathrm{e}}^{\mathrm{TF}}=\sqrt{3 / 8} k_{j}$ (equation (34)).

Finally, the attenuation is obtained with the gain $\left|H\left(\omega_{-}\right)\right|_{\omega_{\mathrm{e}}=\omega_{\mathrm{e}}^{\mathrm{TF}}, \xi_{\mathrm{e}}=\xi_{\mathrm{e}}^{\mathrm{TF}}}$ at point $F_{-}$, without neglecting the structural damping $\xi_{j}$, by symbolic computations. One shows that $A_{\mathrm{dB}}^{\mathrm{RL}}=10 \log f\left(k_{j}, \xi_{j}\right)$ with

$$
f\left(k_{j}, \xi_{j}\right)=\frac{n\left(k_{j}, \xi_{j}\right)}{8\left(6 k_{j}^{2}+8-3 k_{j} \sqrt{2} \sqrt{1+k_{j}^{2}}\right) \xi_{j}^{2}\left(1-\xi_{j}^{2}\right)},
$$

with

$$
\begin{aligned}
& n\left(k_{j}, \xi_{j}\right)=8\left[9 k_{j}^{4}+17 k_{j}^{2}+8-k_{j} \sqrt{2} \sqrt{1+k_{j}^{2}}\left(6 k_{j}^{2}+7\right)\right] \xi_{j}^{2} \\
& +16 \sqrt{3} k_{j}\left(1+k_{j}^{2}\right)\left(\sqrt{2} \sqrt{1+k_{j}^{2}}-k_{j}\right) \xi_{j} \\
& \quad-3 k_{j}^{3} \sqrt{2}\left(1+k_{j}^{2}\right)^{3 / 2}+6 k_{j}^{6}+14 k_{j}^{4}+8 k_{j}^{2} .
\end{aligned}
$$

One can verify that $\xi_{j}=0$ in the above expression leads to expression (A.14).

\section{Appendix B. Synthetic inductor}

The modified version of the Antoniou synthetic inductor proposed in [48] is used and shown in figure B.1. Its equivalent electric impedance is

$$
\begin{aligned}
& Z(\Omega)=\frac{V}{I}=\mathrm{j} L_{\mathrm{eq}} \Omega-R_{\mathrm{eq}}, \quad \text { with } L_{\mathrm{eq}}=\frac{C R_{3} R_{1}}{R_{2}} P_{2}, \\
& R_{\text {eq }}=\frac{R_{1}}{R_{2}} P_{1} .
\end{aligned}
$$

$P_{2}$ and $P_{1}$ are two resistive potentiometers that enable us to tune the equivalent inductance $L_{\mathrm{eq}}$ and a negative resistance
$R_{\text {eq }}$. In our experiments, $R_{\text {eq }}$ has been set to zero, since no parasitic resistance had to be canceled. The amplifiers used were type OPA $445 \mathrm{AP}$, selected for their relatively high operating voltage [49]. Care must be taken regarding ground loops and shorts, as this inductor is grounded and the structure may or may not be isolated from the ground and circuit, particularly in the series shunt case.

\section{References}

[1] Hurlebaus S and Gaul L 2006 Adaptive structures-an overview IMAC XXIV: 24th Int. Modal Analysis Conf. (St. Louis)

[2] Ayela C, Nicu L, Soyer C, Cattan É and Bergaud C 2006 Determination of the $d_{31}$ piezoelectric coefficient of $\mathrm{PbZr}_{x} \mathrm{Ti}_{1-x} \mathrm{O}_{3}$ thin films using multilayer buckled micromembranes J. Appl. Phys. 100054908

[3] Li H, Piekarski B, DeVoe D L and Balachandran B 2008 Nonlinear oscillations of piezoelectric microresonators with curved cross-sections Sensors Actuators A 144 194-200

[4] Karabalin R B, Matheny M H, Feng X L, Defaÿ E, Le Rhun G, Marcoux C, Hentz S, Andreucci P and Roukes M L 2009 Piezoelectric nanoelectromechanical resonators based on aluminum nitride thin film Appl. Phys. Lett. 95103111

[5] Hagood N W and Von Flotow A 1991 Damping of structural vibrations with piezoelectric materials and passive electrical networks J. Sound Vib. 146 243-68

[6] Forward R L 1979 Electronic damping of vibrations in optical structures Appl. Opt. 18 690-7

[7] Snowdon J C 1968 Vibration and Shock in Damped Mechanical Systems (New York: Wiley)

[8] Caruso G 2001 A critical analysis of electric shunt circuits employed in piezoelectric passive vibration damping Smart Mater. Struct. 10 1059-68

[9] Park C H 2003 Dynamics modelling of beams with shunted piezoelectric elements J. Sound Vib. 268 115-29

[10] Behrens S, Moheimani S O R and Fleming A J 2003 Reducing the inductance requirements of piezoelectric shunt damping systems Smart Mater. Struct. 12 57-64

[11] Tang J and Wang K W 2001 Active-passive hybrid piezoelectric networks for vibration control: comparisons and improvement Smart Mater. Struct. 10 794-806

[12] de Marneffe B and Preumont A 2008 Vibration damping with negative capacitance shunts: theory and experiment Smart Mater. Struct. 17035015

[13] Hollkamp J J 1994 Multimodal passive vibration suppression with piezoelectric materials and resonant shunts J. Intell. Mater. Syst. Stuct. 5 49-57

[14] Alessandroni S, dell Isola F and Porfiri M 2002 A revival of electric analogs for vibrating mechanical systems aimed to their efficient control by pzt actuators Int. J. Solids Struct. 39 5295-324

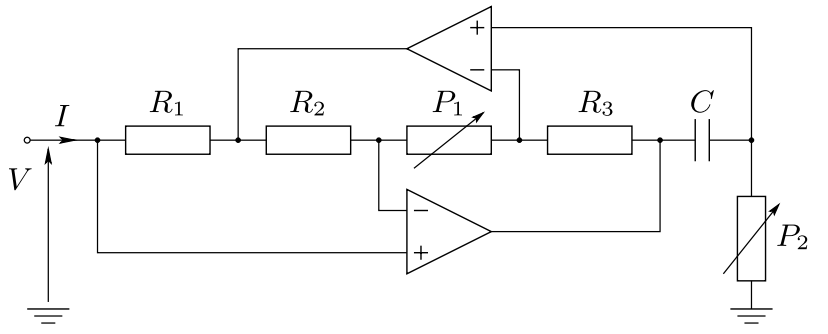

\begin{tabular}{ll}
\hline$R_{1}$ & $2 \mathrm{k} \Omega$ \\
$R_{2}$ & $1 \mathrm{k} \Omega$ \\
$R_{3}$ & $1 \mathrm{k} \Omega$ \\
$P_{1}$ & 0 to $1 \mathrm{k} \Omega$ \\
$C_{1}$ & $10 \mu \mathrm{F}$ \\
$P_{2}$ & External potentiometers \\
\hline
\end{tabular}

Figure B.1. The modified Antoniou synthetic inductor. 
[15] dell'Isola F, Maurini C and Porfiri M 2004 Passive damping of beam vibrations through distributed electric networks and piezoelectric transducers: prototype design and experimental validation Smart Mater. Struct. 13 299-308

[16] Maurini C, dell'Isola F and Del Vescovo D 2004 Comparison of piezoelectronic networks acting as distributed vibration absorbers Mech. Syst. Signal Process. 18 1243-71

[17] Collet M, Cunefare K A and Ichchou M N 2009 Wave motion optimization in periodically distributed shunted piezocomposite beam structures J. Intell. Mater. Syst. Struct. 20 787-808

[18] Casadei F, Ruzzene M, Dozio L and Cunefare K A 2010 Broadband vibration control through periodic arrays of resonant shunts: experimental investigation on plates Smart Mater. Struct. 19015002

[19] Hollkamp J J and Starchville T F Jr 1994 A self-tuning piezoelectric vibration absorber J. Intell. Mater. Syst. Struct. 5 559-66

[20] Niederberger D, Fleming A, Moheimani S O R and Morari M 2004 Adaptive multi-mode resonant piezoelectric shunt damping Smart Mater. Struct. 13 1025-35

[21] Tsai M S and Wang K W 1999 On the structural damping characteristics of active piezoelectric actuators with passive shunt J. Sound Vib. 221 1-22

[22] Schoeftner J and Irschik H 2009 Passive damping and exact annihilation of vibrations of beams using shaped piezoelectric layers and tuned inductive networks Smart Mater. Struct. 18125008

[23] Ducarne J, Thomas O and Deü J-F 2010 Structural vibration reduction by switch shunting of piezoelectric elements: modelling and optimization J. Intell. Mater. Syst. Struct. $21797-816$

[24] Lesieutre G A 1998 Vibration damping and control using shunted piezoelectric materials Shock Vib. Dig. 30 187-95

[25] Moheimani S O 2003 A survey of recent innovations in vibration damping and control using shunted piezoelectric transducers IEEE Trans. Control Syst. Technol. 11 482-94

[26] Leo D J 2007 Engineering Analysis of Smart Material Systems (New York: Wiley)

[27] Sun H, Yang Z, Li K-X, Li B, Xie J, Wu D and Zhang L-L 2009 Vibration suppression of a hard disk driver actuator arm using piezoelectric shunt damping with a topology-optimized pzt transducer Smart Mater. Struct. 18065010

[28] ANSI/IEEE Std 176-1987 1988 IEEE Standard on Piezoelectricity (Piscataway, NJ: The Institute of Electrical and Electronics Engineers, Inc.)

[29] Davis C L and Lesieutre G A 1995 A modal strain energy approach to the prediction of resistively shunted piezoceramic damping J. Sound Vib. 184 129-39

[30] Lesieutre G A and Davis C L 1997 Can a coupling coefficient of a piezoelectric device be higher than those of its active material? J. Intell. Mater. Syst. Struct. 8 859-67

[31] Trindade M A and Benjeddou A 2009 Effective electromechanical coupling coefficients of piezoelectric adaptive structures: critical evaluation and optimization Mech. Adv. Mater. Struct. 16 210-23

[32] Sénéchal A, Thomas O and Deü J-F 2010 Optimization of shunted piezoelectric patches for vibration reduction of complex structures - application to a turbojet fan blade Proc. ASME 2010 Int. Design Engineering Technical Conf. \& Computers and Information in Engineering Conf., IDETC/CIE 2010 (Montreal, August)

[33] Bisegna P, Caruso G and Maceri F 2006 Optimized electric networks for vibration damping of piezoactuated beams J. Sound Vib. 289 908-37

[34] Maurini C, Porfiri M and Pouget J 2006 Numerical methods for modal analysis of stepped piezoelectric beams $J$. Sound Vib. 298 918-33

[35] Ducarne J, Thomas O and Deü J-F 2011 Optimization of piezoelectric shunts for vibration reduction J. Sound Vib. submitted

[36] Piefort V and Preumont A 2001 Finite element modeling of piezoelectric structures Samtech User's Conference (Paris, January) www.ulb.ac.be/scmero/piezo.html

[37] Becker J, Fein O, Maess M and Gaul L 2006 Finite element-based analysis of shunted piezoelectric structures for vibration damping Comput. Struct. 84 2340-50

[38] Collet M and Cunefare K A 2008 Modal synthesis and dynamical condensation methods for accurate piezoelectric systems impedance computation J. Intell. Mater. Syst. Struct. 19 1251-69

[39] Thomas O, Deü J-F and Ducarne J 2009 Dynamics of an elastic structure with piezoelectric patches: finite-element formulation and electromechanical coupling coefficients Int. J. Numer. Methods Eng. 80 235-68

[40] den Hartog J P 1956 Mechanical Vibrations (New York: McGraw Hill) (reprinted by Dover)

[41] Huntsman 2010 Araldite 2011 www.huntsman.com

[42] Vishay Micro-Measurements 2010 Strain Gage Installation with M-Bond 200 Adhesive www.vishaypg.com/micro-measurements

[43] Ducarne J 2009 Modélisation et optimisation de dispositifs non linéaires d'amortissement de structures par systèmes piézoélectriques (Modeling and optimisation of non-linear vibration damping by switch shunting of piezoelectric elements) PhD Thesis Conservatoire National des Arts et Métiers, Paris (in French) http://tel.archives-ouvertes.fr/tel-00464513/fr/

[44] Thomas O, Touzé C and Chaigne A 2003 Asymmetric non-linear forced vibrations of free-edge circular plates, part 2: experiments J. Sound Vib. 265 1075-101

[45] Polytec PI 2008 Piezoelectric Ceramic Products www.piceramic.de/pdf/KATALOG_english.pdf

[46] Brock J E 1946 A note on the damped vibration absorber J. Appl. Mech. 13 A-284

[47] Liu K and Liu J 2005 The damped dynamic vibration absorbers: revisited and new result J. Sound Vib. 284 1181-9

[48] von Vangenheim L 1996 Modification of the classical gic structure and its application to rc-oscillators Electron. Lett. 32 6-8

[49] Texas Instruments 2010 OPA445, High Voltage FET-Input Operational Amplifier http://focus.ti.com/docs/prod/folders/print/opa445.html 